\title{
Geometrische Theoreme.
}

(Bruchstücke aus den hinterlassenen Papieren von C. G. J. Jacobi, mitgetheilt durch Herrn O. Hermes.)

\section{(Vorbemerkung von Herrn 0 . Hermes.)}

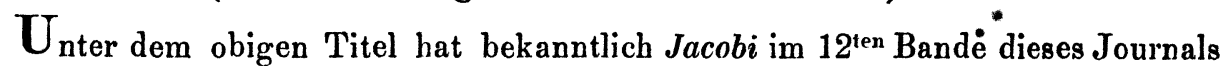
in einem Briefe an Steiner die ersten Mittheilungen über die nach ihm benannte Erzeugungsweise der Linien und Flächen zweiten Grades gemacht. Die vorliegenden Bruchstücke handeln über denselben Gegenstand, und obschon nur in losem Zusammenhange, gestatten sie doch eine Einsicht in die Methode, welcher sich Jacobi bei der Bearbeitung des erwähnten Themas bedient; sie sind bis vor einiger Zeit getrennt in verschiedenen Händen gewesen, und es hat darum ihre Herausgabe einen so langen Aufschub erlitten. Diesem Umstande ist es vorzüglich zuzuschreiben, dass die Jacobische Ausdehnung der Focalerzeugung der Kegelschnitte auf die Flächen des zweiten Grades, obgleich die einfachste, anschaulichste und vollständigste von allen Erzeugungsweisen dieser. Flächen, noch keineswegs diejenige Anerkennung gefunden hat, welche sie in so hohem Maasse verdient, und dass man immer noch das Ivorysche Theorem über die confocalen Kegelschnitte und Flächen zweiten Grades, von welchem Jacobi bei der Entwicklung seiner Sätze ausgeht, als „für die Geometrie noch nicht genug ausgebeutet" bezeichnen muss.

In dem ersten Bruchstücke, welches von Jacobi selbst die Ueberschrift „Geometrische Theoreme" erhalten hat, findet sich nach einer kurzen Einleitung, in welcher $J a c o b i$ eine genauere Discussion der erzeugten Curven und Flächen zu liefern verspricht, eine kurze analytische Darstellung seiner Methode, die Focaleigenschaften der Kegelschnitte aus dem Ivoryschen Theorem herzuleiten. Das zweite, umfangreichere Bruchstück enthält die Lösung einer Aufgabe über confocale Kegelschnitte, welche für die spätere Discussion der entstehenden Flächen eine nothwendige Voraussetzung bildet, und an diese sich anschliessend diese Discussion selbst und eine Verallgemeinerung der Entstehungsweise der Flächen.

Diese letzten Untersuchungen erscheinen ebenfalls nur in einem ziemlich losen Zusammenhange mit einander; jedoch ist absichtlich der Wortlaut des Manuscriptes so treu als möglich beibehalten worden. Zur Erleichterung des Verständnisses hat sich der Herausgeber veranlasst gesehen, jedes der beiden Bruchstücke, aus welchen sich diese nachgelassene Jacobische Arbeit zusammensetzt, durch eine Vorbemerkung einzuleiten. Noch ist zu erwähnen, dass der grössere Theil des zweiten Bruchstücks bereits von dem im Frühjahr 1861 verstorbenen Dr. Sigismund Cohn geordnet und für den Druck vorbereitet worden ist.

\section{Erstes Bruchstück.}

Wenn man über einer festen Basis Dreiecke errichtet, deren beide Schenkel eine constante Summe oder eine constante Differenz haben, so ist der Ort der Spitze dieser Dreiecke ein Kegelschnitt. Man kann diesen Satz auch so aussprechen: 
Wenn man über zwei festen Basen $A B$ und $C D$ mit denselben Schenkelpaaren $A P=C Q, B P=D Q$ Dreiecke errichtet, und die einen Dreiecke $C D Q$ verschwinden, so ist der Ort der Spitzen $P$ der anderen Dreiecke ABP ein Kegelschnitt.

Denn wenn ein Dreieck verschwindet, so fällt die Spitze in die Grundlinie, und es wird die Summe oder die Differenz der Schenkel gleich der Grundlinie selber, also constant. Während bei der gewöhnlichen Art, den angeführten Elementarsatz auszudrücken, sich nicht absehen liess, wie er auf Flächen zoveiter Ordnung ausgedehnt werden kann, so bietet diese neue Art, ihn auszusprechen, den Vortheil dar, die verlangte Verallgemeinerung unmittelbar und auf die natürlichste Weise zu geben. Man hat nämlich den ganz analogen Satz für den Raum:

Wenn man über zwei festen Dreiecken $A B C$ und $D E F$ als Basen mit denselben Schenkeln $A P=D Q, B P=E Q, C P=F Q$ Pyramiden errichtet, und die uber der einen Basis DEF errichteten Pyramiden DEFQ verschwinden, so ist der Ort der Spitze $P$ der über der anderen Basis errichteten Pyramiden $A B C P$ eine Fläche zweiter Ordnung.

Wenn die Pyramide DEFQ verschwindet, so fällt die Spitze $Q$ in die Ebene der Basis DEF. Man hat aber, auch die allgemeineren Saatze, dass der Ort der Spitze $P$ eine Curve oder Fläche zweiter Ordnung ist, wenn $Q$ in einer beliebig gegebenen geraden Linie oder Ebene liegt.

Ich habe diese Sätze zuerst im 12 $2^{\text {ten }}$ Bande des Crelleschen Journals (J. 1834) in einem Briefe an Steiner mitgetheilt. Da sie eine Lücke in der Theorie der Flächen zweiter Ordnung auszufüllen scheinen, so will ich sie hier näher erörtern. Ich werde zuerst zeigen, wie sie eine unmittelbare Folge des für die Geometrie noch nicht genug ausgebeuteten Theorems Ivorys sind, dass die Verbindungslinie beliebiger zwei in confocalen Ellipsen oder Ellipsoiden liegender Punkte gleich der. Verbindungslinie der beiden ihnen in denselben Ellipsen oder Ellipsoiden conjugirten Punkte ist.

Hierauf werde ich in eine genauere Discussion der erzeugten Curven oder Flächen eingehen. Ich beginne mit den Kegelschnitten.

Es sei die Gleichung einer gegebenen Ellipse, auf ihre Hauptachsen bezogen,

$$
\frac{x x}{m m}+\frac{y y}{n n}=1
$$


und die Gleichung einer ihr confocalen Ellipse

$$
\frac{x x}{m m+\varrho}+\frac{y y}{n n+\varrho}=1,
$$

wo $n<m$ sein mag. Zwei Punkte dieser Ellipsen, $P$ und $Q$, deren Coordinaten

und

$$
x=m \alpha, \quad y=n \beta
$$

sind, wo

$$
x=\sqrt{m m+\varrho} \cdot \alpha, \quad y=\sqrt{n n+\varrho} \cdot \beta
$$

$$
\alpha \alpha+\beta \beta=1,
$$

heissen einander conjugirt. Hat man zwei andere conjugirte Punkte $\boldsymbol{P}^{\prime}$ und $Q^{\prime}$, deren Coordinaten

und

$$
x=m \alpha^{\prime}, \quad y=n \beta^{\prime}
$$

$$
x=\sqrt{m m+\varrho} \cdot \alpha^{\prime}, \quad y=\sqrt{n n+\varrho} \cdot \beta^{\prime}
$$

sind, wo wieder

so wird

$$
\alpha^{\prime} \alpha^{\prime}+\beta^{\prime} \beta^{\prime}=1,
$$

$$
\begin{aligned}
& P Q^{\prime 2}=\left(m \alpha-\sqrt{m m+\varrho} \cdot \alpha^{\prime}\right)^{2}+\left(n \beta-\sqrt{n n+\varrho} \cdot \beta^{\prime}\right)^{2}, \\
& P^{\prime} Q^{2}=\left(\sqrt{m m+\varrho} \cdot \alpha-m \alpha^{\prime}\right)^{2}+\left(\sqrt{n n+\varrho} \cdot \beta-n \beta^{\prime}\right)^{2}
\end{aligned}
$$

und daher

$$
P Q^{\prime 2}-P^{\prime} Q^{2}=\rho\left(\alpha^{\prime} \alpha^{\prime}+\beta^{\prime} \beta^{\prime}-\alpha \alpha-\beta \beta\right)=0
$$

oder

$$
P Q^{\prime}=P^{\prime} Q,
$$

welches der Ivorysche Satz für Ellipsen ist. Setzt man gleichzeitig $n \sqrt{ }-1$ und $\beta \sqrt{ }-1$ für $n$ und $\beta$, so erhält man denselben Satz für confocale Hyperbeln.

Lässt man $\varrho$ bis zu dem Werthe - nn abnehmen, und $n n+\rho$ und $y$ gleichzeitig verschwinden, so hat man für die Punkte der zweiten Ellipse

$$
x=\sqrt{m m-n n} . \alpha, \quad y=0 .
$$

Dieselbe reducirt sich daher, da $\alpha$ immer zwischen -1 und +1 liegt, auf das zwischen den beiden Brennpunkten gelegene Stück der grossen Achse, welches ich die Grensellipse nennen werde. Es seien $0, F, F^{\prime}$ der Mittelpunkt und die Brennpunkte der gegebenen Ellipse, $A$ und $A^{\prime}$ die Endpunkte ihrer grossen, $B$ und $B^{\prime}$ die Endpunkte ihrer kleinen Achse. Setzt man $\alpha=0, \beta= \pm 1$, so erhält man als die conjugirten Punkte den Punkt $O$ der Grenzellipse und die Punkte $B$ oder $B^{\prime}$ der gegebenen Ellipse. Setzt man dagegen $\alpha= \pm 1, \beta=0$, so 
sieht man, dass den Punkten $F$ und $F^{\prime}$ der Grenzellipse die Punkte $A$ und $A^{\prime}$ der gegebenen conjugirt sind. Sind daher allgemein $P$ und $Q$ zwei beliebige conjugirte Punkte der gegebenen Ellipse und der Grenzellipse, so hat

Fig. 1.

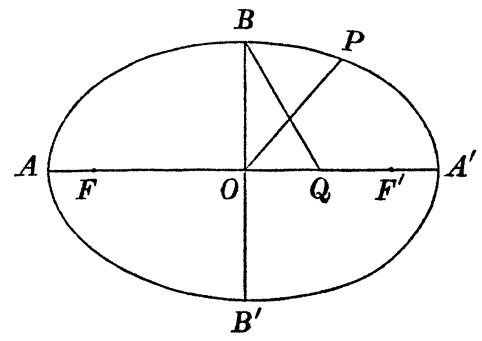

man nach dem Ivoryschen Theorem

$P F=Q A, \quad P F^{\prime}=Q A^{\prime}, \quad P O=Q B=Q B^{\prime}$

und daher

d. $\mathrm{h}$.

$$
P F+P F^{\prime}=A A^{\prime},
$$

die Summe der von den beiden Brennpunten einer Ellipse $F$ und $F^{\prime}$ nach einem ihrer

Punkte $P$ gezogenen Radii vectores ist immer der grossen Achse AA' gleich, und wenn man auf dieser die den beiden Radii vectores gleichen Stücke $A Q=F P, Q A^{\prime}=P F^{\prime}$ abträgt, so wird die Entfernung des Punktes $Q$ von den Endpunkten der kleinen Achse gleich der Entfernung des Punktes. $P$ vom Mittelpunkt der Ellipse.

Wenn durch die Gleichung

$$
\frac{x x}{m m}-\frac{y y}{n n}=1
$$

eine Hyperbel gegeben ist, deren Mittelpunkt, Brennpunkte und Endpunkte der grossen (d.h. reellen) Achse wieder $O, F$ und $F^{\prime}$, und $A$ und $A^{\prime}$ heissen mögen, so sind die ihr confocalen Hyperbeln in der Gleichung

$$
\frac{x x}{m m+\varrho}-\frac{y y}{n n-\varrho}=1
$$

enthalten. Zwei conjugirte Punkte $P$ und $Q$ der gegebenen und einer ihr confocalen Hyperbel haben zu Coordinaten

$$
\begin{gathered}
x=m \alpha, \quad y=n \beta, \\
x=\sqrt{m m+\varrho} \cdot \alpha, \quad y=\sqrt{n n-\varrho} \cdot \beta,
\end{gathered}
$$

wo

$$
\alpha \alpha-\beta \beta=1 .
$$

Eine Grenze der confocalen Hyperbeln entspricht dem Werthe $\varrho=n n$. Lässt man $y$ und $n n-\varrho$ gleichzeitig verschwinden, so wird für die Punkte dieser Grenzhyperbel

$$
x=\sqrt{m m+n n} . \alpha, \quad y=0 .
$$

Es reducirt sich daher diese Hyperbel, da $\alpha>1$, auf die über $F$ und $F^{\prime}$ hinaus nach beiden Seiten ins Unendliche gehende Verlängerung der Linie $F F^{\prime}$. 
Setzt man $\alpha= \pm 1$, so sieht man, dass den Punkten $F$ und $F^{\prime}$ der Grenzhyperbel die Scheitel $\boldsymbol{A}$ und $\boldsymbol{A}^{\prime}$ der gegebenen Hyperbel conjugirt werden. Wenn der Punkt $Q$, von $F$ oder $F^{\prime}$ an, die unendliche Verlängerung von $\boldsymbol{F} F^{\prime}$ über $F$ oder $F^{\prime}$ hinaus durchläuft, bewegt sich $\boldsymbol{P}$ auf der einen Hälfte des einen Zweiges der Hyperbel, und in jedem Augenblicke hat man

$$
P F=Q A, \quad P F^{\prime}=Q A^{\prime},
$$

also

$$
P F^{\prime}-P F=Q A^{\prime}-Q A=A A^{\prime},
$$

welches der bekannte Satz für die Hyperbel ist.

Man erhält für die confocalen Hyperbeln eine zweite Grenze, welche dem Werthe $\varrho=-m m$ entspricht. Lässt man nämlich $x$ und $m m+\varrho$ gleichzeitig verschwinden, so wird für die Punkte dieser Grenzhyperbel

$$
x=0, \quad y=\sqrt{m m+n n} . \beta,
$$

und da $\beta$ keiner Begrenzung unterworfen ist, so reducirt sie sich auf die ganze Länge der kleinen (d. h. imaginären) Achse. Setzt man $\beta=0$, so sieht man, dass dem Punkte $O$ dieser Grenzhyperbel die Scheitel (Endpunkte der grossen Achse) der gegebenen Hyperbel entsprechen ${ }^{*}$ ).

Hat man daher auf der gegebenen Hyperbel den Punkt $\boldsymbol{P}$ und auf der kleinen Achse den conjugirten Punkt $Q$ der zweiten Grenzhyperbel, so wird $A Q=O P$, wodurch man leicht $Q$ findet, wenn $\boldsymbol{P}$ gegeben ist und umgekehrt. Man wird deshalb aus dem Ivoryschen Theorem folgenden Satz ableiten können:

Wenn man aus dem Mittelpunkte $O$ und dem Scheitel $A$ einer Hyperbel respective nach

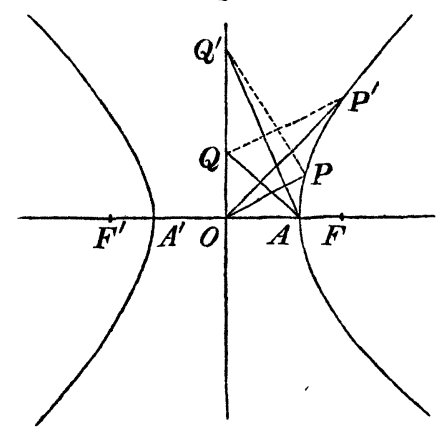
der Hyperbel und der kleinen Achse die gleichen Linien

$$
O P=A Q, \quad O P^{\prime}=A Q^{\prime}
$$

zieht, so hat man auch $P Q^{\prime}=P^{\prime} Q$.

Das Ivorysche Theorem führt aber mit derselben Leichtigkeit auf einen allgemeineren Satz, welcher eine Eigenschaft der beiden Linien angiebt, die

*) Man könnte auch so die beiden Achsen und mithin jede zwei zu einander rechtwinkligen Linien auf einander beziehen und immer zwei Punkte auf ihnen als conjugirte betrachten, wenn die Quadrate ihrer Abstände rom Schnittpunkte eine constante Differenz haben. 
man aus den Punkten eines Kegelschnitts, z. B. der Ellipse Fig. 3, nach zwei be-

Fig. 3.

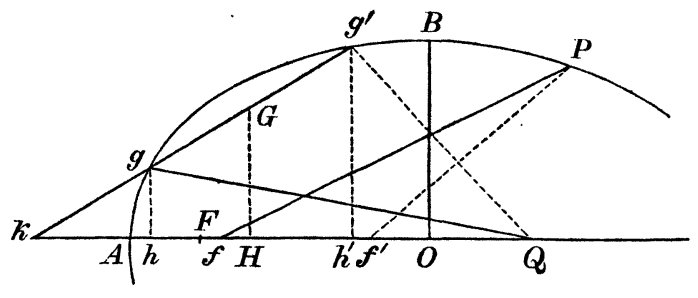

liebigen festen Punkten der grossen Achse zieht. Es seien nämlich $f$ und $f^{\prime}$ zwei beliebige feste Punkte der Linie $F F^{\prime}$, die Punkte $g$ und $g^{\prime}$ ihnen auf der Ellipse conjugirt, so hat man für jeden Punkt $Q$ der Linie und den inm conjugirten $P$ der Ellipse

und daher folgenden Satz:

$$
P f=Q g, \quad P f^{\prime}=Q g^{\prime}
$$

\section{Satz $I$.}

Die beiden Linien, die man von zwei auf der grossen Achse zwischen den Brennpunkten beliebig liegenden festen Punkten nach den verschiedenen Punkten einer Ellipse zieht, können zu Schenkeln eines über einer anderen festen Basis beschriebenen Dreiecks genommen verden, dessen Spitze eine gerade Linie durchläuft.

Man kann diesen Satz auch so darstellen, wie ich ihn am angeführten Orte (Cr. J. Bd. XII) ausgesprochen habe:

\section{Satz II.}

Wenn man über zwei festen Basen $g g^{\prime}$ und $f^{\prime}$ mit denselben Schenkeln $Q g=P f, Q g^{\prime}=P f^{\prime}$ Dreiecke beschreibt, und die Spitze $Q$ der einen Dreiecke eine beliebig gegebene gerade Linie durchläuft, so beschreibt die Spitze P der anderen Dreiecke einen Kegelschnitt.

Wenn die gegebene gerade Linie die Basis $g^{\prime}$ selber ist, so werden $f, f^{\prime}$ die Brennpunkte des Kegelschnitts. Aber man hat auch den allgemeineren Satz, dass, wenn die gerade Linie durch den einen Endpunkt $g$ der Basis geht, der entsprechende Endpunkt f der anderen Basis der eine Brennpunkt des Kegelschnitts wird. Denn, wenn der Punkt $g$ des Kegelschnitts in die gerade Linie $f f^{\prime}$, die grosse Achse, zu liegen kommt, so fällt $g$ mit dem Endpunkt der grossen Achse $A$ zusammen und der conjugirte Punkt $f$ mit dem Brennpunkt $F$.

Ich will jetzt den nach Satz II. erzeugten Kegelschnitt näher bestimmen, wobei ich der besseren Unterscheidung wegen die Linien $g g^{\prime}$ und $f f^{\prime}$ die erste und zweite Basis nennen werde. Es sei in der ursprünglichen Figur 3, welche dem Satz I. zu Grunde liegt,

$$
O f=\sqrt{m m-n n} \cdot \alpha, \quad O f^{\prime}=\sqrt{m m-n n} . \alpha^{\prime},
$$


so wird die zweite Basis

$$
f f^{\prime}=\sqrt{m m-n n}\left(\alpha-\alpha^{\prime}\right) .
$$

Die den Punkten $f$ und $f^{\prime}$ conjugirten Punkte des Kegelschnitts $g$ und $g^{\prime}$ haben die Coordinaten

wo

$$
m \alpha, \quad m \beta \text { und } m \alpha^{\prime}, \quad m \beta^{\prime} \text {, }
$$

$$
\alpha \alpha+\beta \beta=\alpha^{\prime} \alpha^{\prime}+\beta^{\prime} \beta^{\prime}=1 \text {. }
$$

Sind $h$ und $h^{\prime}$ die Fusspunkte der von $g$ und $g^{\prime}$ auf die gerade Linie gefällten Perpendikel, so sind $O h=m \alpha, O h^{\prime}=m \alpha^{\prime}$ gegeben, und wenn $H$ die Mitte von $h$ und $h^{\prime}$ ist,

$$
2 O H=m\left(\alpha+\alpha^{\prime}\right),
$$

ferner die Projection der ersten Basis auf diese Linie

und daher

$$
h h^{\prime}=m\left(\alpha-\alpha^{\prime}\right)
$$

$$
\frac{\sqrt{m m-n n}}{m}=\frac{f f^{\prime}}{h h^{\prime}}, \quad \frac{n}{m}=\frac{\sqrt{h h^{\prime 2}-f f^{\prime 2}}}{h h^{\prime}} .
$$

Ueberdies hat man

und daher

$$
g h=n \beta, \quad g^{\prime} h^{\prime}=n \beta^{\prime}
$$

woraus

$$
\frac{h h^{\prime 2}\left(g^{\prime} h^{\prime 2}-g h^{2}\right)}{h h^{\prime 2}-f f^{\prime 2}}=m^{2}\left(\beta^{\prime 2}-\beta^{2}\right)=m^{2}\left(\alpha^{2}-\alpha^{\prime 2}\right)=m\left(\alpha+\alpha^{\prime}\right) \cdot h h^{\prime},
$$

$$
2 O H=O h+O h^{\prime}=\frac{h h^{\prime}\left(g^{\prime} h^{2}-g h^{2}\right)}{h h^{2}-f f^{\prime 2}} .
$$

Diese Gleichung bestimmt die Lage des Mittelpunkts $O$ des Kegelschnitts. Hat man $O$ gefunden, so zeigen die Gleichungen

$$
O f=\frac{f f^{\prime}}{h h^{\prime}} \cdot O h, \quad O f^{\prime}=\frac{f f^{\prime}}{h h^{\prime}} \cdot O h^{\prime},
$$

wo man die Punkte $f$ und $f^{\prime}$ in der gegebenen geraden Linie anzunehmen hat, damit die ursprüngliche, dem Satz I. zu Grunde liegende Figur 3 erzeugt wird, in welcher der erzeugte Kegelschnitt die gegebene gerade Linie zur grossen Achse hat und durch die festen Punkte $g$ und $g^{\prime}$ geht, die den Punkten $f$ und $f^{\prime}$ conjugirt werden.

Man kann den für $\mathrm{OH}$ gefundenen Ausdruck etwas einfacher darstellen, wenn man den Schneidungspunkt $k$ der nöthigenfalls verlängerten ersten Basis $g g^{\prime}$ mit der gegebenen geraden Linie zu Hilfe nimmt. Man hat nämlich

$$
k h: k h^{\prime}=g h: g^{\prime} h^{\prime}
$$


und daher

Da

$$
k h^{\prime}+k h: h h^{\prime}=g^{\prime} h^{\prime}+g h: g^{\prime} h^{\prime}-g h
$$

so folgt hieraus

$$
k h^{\prime}+k h=2 k H,
$$

$$
\begin{gathered}
O H=k H \cdot \frac{\left(g^{\prime} h^{\prime}-g h\right)^{2}}{h h^{\prime 2}-f f^{\prime 2}}=k H \cdot \frac{g g^{\prime 2}-h h^{\prime 2}}{h h^{\prime 2}-f f^{\prime 2}}, \\
k O=k H \cdot \frac{g g^{\prime 2}-f f^{\prime 2}}{h h^{\prime 2}-f f^{\prime 2}} .
\end{gathered}
$$

Man findet ferner nach einiger Rechnung, wenn $G$ der Mittelpunkt der ersten Basis $g g^{\prime}$ gegeben ist, das Quadrat der halben kleinen Achse

$$
n^{2}=\frac{\left(g g^{\prime 2}-f f^{\prime 2}\right)\left(G h^{2}-\frac{1}{4} f f^{\prime 2}\right)}{h h^{\prime 2}-f f^{\prime 2}}
$$

oder

$$
n^{2}=\frac{k O}{k H} \cdot\left(G h^{2}-\frac{1}{4} f f^{\prime 2}\right)
$$

Aus den gefundenen Formeln leitet man folgende Resultate ab:

I. Wenn die zweite Basis $\mathrm{ff}^{\prime}$ sich auf einen Punkt reducirt, so wird der Kegelschnitt ein um diesen Punkt als Centrum beschriebener Kreis. Die Bedingung, dass der Kegelschnitt eine Ellipse sei, ist

$$
f f^{\prime}<h h^{\prime} \text {. }
$$

Der Mittelpunkt der Ellipse $O$ liegt auf der Verlängerung von $k H$ über $H$ hinaus, und zwar liegen, wenn, wie in Figur 3., $g^{\prime} h^{\prime}>g h$ ist, $h$ und $h^{\prime}$ auf derselben Seite oder auf verschiedenen Seiten von $O$, jenachdem

d. h. jenachdem

$$
g^{\prime} h^{\prime 2}-g h^{2}>\text { oder }<h h^{\prime 2}-f f^{\prime 2}
$$

$$
g^{\prime} h^{\prime 2}+f f^{\prime 2}>\text { oder }<g h^{\prime 2}
$$

II. Wenn $f f^{\prime}=h h^{\prime}$ oder die zweite Basis gleich der Projection der ersten Basis auf die gegebene gerade Linie, so wird der Kegelschnitt eine Parabel.

III. Ist $f f^{\prime}>h h^{\prime}$, so wird der Kegelschnitt eine Hyperbel. Aus den Gleichungen

$$
\begin{aligned}
n^{2} & =\frac{\left(g g^{\prime 2}-f f^{\prime 2}\right)\left(G h^{2}-1 f f^{\prime 2}\right)}{h h^{\prime 2}-f f^{\prime 2}}, \\
m^{2} & =\frac{h h^{\prime 2}\left(g g^{\prime 2}-f f^{\prime 2}\right)\left(G h^{2}-1 f f^{\prime 2}\right)}{\left(h h^{\prime 2}-f f^{\prime 2}\right)^{2}}
\end{aligned}
$$


folgt, dass $n^{2}$ negativ, $m^{2}$ positiv, oder dass die gegebene Linie die grosse Achse der Hyperbel ist, wenn $f^{\prime}$ gleichzeitig kleiner oder grösser ist als die beiden Linien $g g^{\prime}$ und $2 G h$; ferner dass $n^{2}$ positiv, $m^{2}$ negativ, oder dass die gegebene Linie die kleine Achse der Hyperbel ist, wenn $f^{\prime}$ zwischen $g g^{\prime}$ und $2 G h$ enthalten ist. Es ist übrigens

$$
\begin{gathered}
g g^{\prime 2}=h h^{\prime 2}+\left(g^{\prime} h^{\prime}-g h\right)^{2}, \\
4 G h^{2}=h h^{\prime 2}+\left(g^{\prime} h^{\prime}+g h\right)^{2}
\end{gathered}
$$

und daher $g g^{\prime}<$ oder $>2 G h$, jenachdem die Punkte $g$ und $g^{\prime}$ auf derselben oder auf verschiedenen Seiten der gegebenen Linie liegen.

\section{(Vorbemerkung zum zweiten Bruchstück von Herrn O. Hermes.)}

Zur Vermittelung der Schlussfolgerungen, welche Jacobi an die in dem Folgenden entwickelte Aufgabe anknüpft, ist Einzelnes vorweg in Betracht zu ziehen.

Die Gleichung einer auf ihre Hauptachsen bezogenen Fläche

$$
\text { (1.) } \frac{x^{2}}{m^{2}}+\frac{y^{2}}{n^{2}}+\frac{z^{2}}{p^{2}}=1
$$

stellt ein Ellipsoid, ein einflächiges Hyperboloid oder ein zweiflächiges Hyperboloid dar, jenachdem die Grössen $m^{2}, n^{2}, p^{2}$ sämmtlich positiv, oder zwei derselben positiv und die dritte negativ, oder nur eine einzige positiv und die beiden übrigen negativ sind: ferner werden durch die Gleichung

$$
\text { (2.) } \frac{x^{2}}{m^{2}-\lambda^{2}}+\frac{y^{2}}{n^{2}-\lambda^{2}}+\frac{z^{2}}{p^{2}-\lambda^{2}}=1
$$

bekanntlich für verschiedene positive oder negative Werthe von $\lambda^{2}$ die sämmtlichen Flächen eines der Fläche (1.) confocalen Systems dargestellt, und zwar für die besonderen Annahmen $\lambda^{2}=m^{2},=n^{2}$ oder $=p^{2}$ die einzelnen Grenzfälle, welche durch die (reellen oder imaginären) Focalcurven bestimmt sind.

Wegen der Bedeutung, welche gerade diese Grenzfälle für unsere Aufgabe haben, möge ein Beispiel durchgeführt werden. Nimmt man die Grössen $m^{2}, n^{2}, p^{2}$ als positiv an und zwar $m>n>p$, so stellt die Gleichung (2.) für den Werth $\lambda^{2}=p^{2}$, wenn gleichzeitig $z=0$ ist, weil das Glied $\frac{z^{2}}{p^{2}-\lambda^{2}}$ alsdann den unbestimmten Werth $\frac{0}{0}$ annimmt und als positiv oder negativ betrachtet werden kann, verschiedene Räume der $x y$-Ebene dar. Jenachdem man nämlich, unter Zugrundelegung stetig wachsender Werthe von $\lambda^{2}$, den besonderen Werth $p^{2}$ dieser Grösse als die Reihe der confocalen Ellipsoide, welche den Werthen von $\lambda^{2}<p^{2}$ entsprechen, beschliessend, oder die Reihe der confocalen einflächigen Hyperboloide, welche den Werthen $\lambda^{2}>p^{2}$ und $\left\langle n^{2}\right.$ zugehören, beginnend ansieht, entsprechen der Gleichung (2.) entweder die sämmtlichen Punkte der $x y$-Ebene innerhalb oder ausserhalb der Focalellipse

$$
\frac{x^{2}}{m^{2}-p^{2}}+\frac{y^{2}}{n^{2}-p^{2}}=1, \quad z=0,
$$

so dass man im Anschluss an die Jacobische Einführung der Namen Grenzellipse oder Grenzhyperbel in der Ebene, den durch die Gleichung (2.) für den Fall $\lambda^{2}=p^{2}$ und 
$z=0$ ausgedrückten Theil der $x y$-Ebene Grenzellipsoid oder Grenzhyperboloid nennen kann, jenachdem man dem Gliede $\frac{z^{2}}{p^{2}-\lambda^{2}}$ ein positives oder negatives Zeichen beilegt.

Ebenso werden durch die Gleichung (2.) für die Annahme $\lambda^{2}=n^{2}, y=0$ die sämmtlichen Punkte der $x z$-Ebene dargestellt, und zwar wenn man dem Gliede $\frac{y^{2}}{\lambda^{2}-n^{2}}=\frac{0}{0}$ positive Werthe beilegt, vermöge der Ungleichheit

$$
\frac{x^{2}}{m^{2}-n^{2}}-\frac{\dot{z}^{2}}{n^{2}-p^{2}}<1
$$

die sämmtlichen Punkte innerhalb der Focalhyperbel, und wenn das unbestimmte Glied $\frac{y^{2}}{\lambda^{2}-n^{2}}=\frac{0}{0}$ ein negatives Zeichen erhält, vermöge der Ungleichheit

$$
\frac{x^{2}}{m^{2}-n^{2}}-\frac{z^{2}}{n^{2}-p^{2}}>1
$$

alle Punkte der $x z$-Ebene, welche vom Mittelpunkte aus gerechnet jenseits der Focalhyperbel liegen. Die Annahme endlich $\lambda^{2}=m^{2}, x=0$ ergiebt die sämmtlichen Punkte der $y z$-Ebene, und zwar wenn man das alsdann unbestimmte Glied $\frac{x^{2}}{\lambda^{2}-m^{2}}$ als positiv annimmt, vermöge der Ungleichheit

$$
\frac{y^{2}}{m^{2}-n^{2}}+\frac{z^{2}}{m^{2}-p^{2}}>1
$$

alle Punkte dieser Ebene, während die negativen Werthe des unbestimmten Gliedes $\frac{x^{2}}{\lambda^{2}-m^{2}}$ keine geometrische Bedeutung mehr zulassen.

Wendet man nun das Ivorysche Theorem, „dass die Verbindungslinie beliebiger zwei auf confocalen Flächen zweiten Grades liegender Punkte gleich der Verbindungs. linie der beiden ihnen in denselben Flächen conjugirten Punkte ist", auf die Fläche (1.) und einen der Grenzfälle der Fläche (2.) an und zwar in der Weise, dass man auf der letzteren, z. B. im Innern der Focalellipse, drei beliebige Punkte $A^{\prime}, B^{\prime}, C^{\prime}$ annimmt, welchen auf der Fläche (1.) als conjugirt die Punkte $A, B, C$ zugehören, die ersteren als Eckpunkte der Basis einer dreiseitigen Pyramide $A^{\prime} B^{\prime} C^{\prime} P$, die letzteren als Eckpunkte der Basis einer zweiten dreiseitigen Pyramide $\left.A B C Q^{*}\right)$, deren Spitzen $P$ und $Q$ ebenfalls conjugirte Punkte, bezüglich auf den Flächen (1.) und (2.), sind: wenn jetzt die Spitze $\boldsymbol{Q}$ die Grenzfläche (2.), d. h. die Ebene der Focalellipse, durchläuft, so beschreibt die Spitze $P$ der ersten Pyramide die Fläche (1.). Von dieser Betrachtung ausgehend giebt Jacobi (Cr. J. Bd. XII, pag. 139) seine allgemeine Erzeugungsart der Flächen des zweiten Grades, wie folgt:

,Werden im Raume irgend drei feste Punkte $\boldsymbol{A}^{\prime}, \boldsymbol{B}^{\prime}, \boldsymbol{C}^{\prime}$ und irgend drei andere, jenen beziehlich entsprechende, feste Punkte $A, B, C$ angenommen und werden in Rücksicht auf diese Fundamentalpunkte andere entsprechende Punkte $P, Q$ so bestimmt, dass ihre Abstände von jenen respective gleich sind, $d$. h. dass

$$
P A^{\prime}=Q A, \quad P B^{\prime}=Q B, \quad P C^{\prime}=Q C,
$$

so hat man ein Correlativsystem, worin der Punkt $P$ irgend eine Fläche zweiten Grades beschreibt, wenn der Punkt $Q$ sich in einer Ebene bewegt."

Auf diesen allgemeinen Satz beziehen sich die am Schlusse des zweiten Bruchstücks befindlichen Entwicklungen Jacobis.

Wenn im Besonderen die Eckpunkte der ersten Basis $A^{\prime}, B^{\prime}, C^{\prime}$ auf dem Um. fange eines Focalkegelschnitts, z. B. der Focalellipse, liegen, so sind die Eckpunkte

\footnotetext{
*) Es ist hier die Bezeichnung eingeführt, welche Jacobi in dem zweiten Bruchstilick festhält.
} 
$A, B, C$ der zweiten Basis die conjugirten Punkte auf dem $x y$-Schnitt des Ellipsoids (1.), wenn wir auch hier die Annahme $m>n>p$ festhalten. In der eben angegebenen Erzeugungsweise tritt alsdann nur die Aenderung ein, dass die Ebene, welche der Punkt $Q$ durchläuft, mit der Ebene der Basis $A B C$ zusammenfällt, d. h. dass das Volumen der dreiseitigen Pyramide $A B C Q$ für alle Lagen des Punktes $Q$ verschwindet. Die Eckpunkte $A, B, C$ und $A^{\prime}, B^{\prime}, C^{\prime}$ sind nunmehr zugleich conjugirte Punkte confocaler Kegelschnitte, beispielsweise der beiden Ellipsen

$$
\begin{aligned}
\frac{x^{2}}{m^{2}}+\frac{y^{2}}{n^{2}} & =1, \quad z=0, \\
\frac{x^{2}}{m^{2}-p^{2}}+\frac{y^{2}}{n^{2}-p^{2}} & =1, \quad z=0,
\end{aligned}
$$

und es stimmen die Hauptachsen eines dieser Kegelschnitte der Länge und Lage nach überein mit den Hauptachsen eines Hauptschnitts der durch den Punkt $\boldsymbol{P}$ beschriebenen Fläche (1.), während durch die Differenz der Quadrate der entsprechenden Halbachsen der beiden Kegelschnitte zugleich das Quadrat der zu dem entsprechenden Hauptschnitte senkrechten Halbachse der Fläche (1.) bestimmt wird.

Es kommt demnach die Aufgabe, die Art der von der Spitze $P$ erzeugten Fläche zu bestimmen, wenn die beiden Basen $A B C$ und $A^{\prime} B^{\prime} C^{\prime}$ gegeben sind, und der dem Punkte $\boldsymbol{P}$ conjugirte Punkt, d. h. für welchen nach dem Ivoryschen Theorem

$$
P A^{\prime}=Q A, \quad P B^{\prime}=Q B, \quad P C^{\prime}=Q C,
$$

die Ebene des Dreiecks $A B C$ durchläuft, hinaus auf die Lösung der Aufgabe, mit welcher das folgende Bruchstück beginnt.

\section{Zweites Bruchstück.}

Um zwei beliebige Dreiecke $A B C$ und $A^{\prime} B^{\prime} C^{\prime}$ Kegelschnitte zu beschreiben, welche gleiche Excentricität haben, und in welchen $A$ und $A^{\prime}, B$ und $B^{\prime}, C$ und $C^{\prime}$ conjugirte Punkte sind.

Wenn in zwei ebenen Figuren sich je zwei Punkte so entsprechen, dass ihre Abscissen sich wie $m: m^{\prime}$ und ihre Ordinaten wie $n: n^{\prime}$ verhalten, so verhält sich der Inhalt eines zwischen drei Punkten der einen Figur beschriebenen Dreieckes zum Inhalt des zwischen den entsprechenden Punkten der andern Figur beschriebenen Dreieckes wie $m n: m^{\prime} n^{\prime}$. Sind daher die auf die Hauptachsen bezogenen Gleichungen der beiden gesuchten Kegelschnitte

so hat man:

$$
\frac{x^{2}}{m m}+\frac{y^{2}}{n n}=1, \quad \frac{x^{2}}{n^{\prime} m^{\prime}}+\frac{y^{2}}{n^{\prime} n^{\prime}}=1,
$$

$$
A B C: A^{\prime} B^{\prime} C^{\prime}=m n: m^{\prime} n^{\prime} .
$$

Aus demselben Grunde hat man, wenn man $O$ und $O^{\prime}$ die Mittelpunkte der beiden Kegelschnitte nennt,

$$
\frac{O^{\prime} B^{\prime} C^{\prime}}{O B C}=\frac{O^{\prime} C^{\prime} A^{\prime}}{O C A}=\frac{O^{\prime} A^{\prime} B^{\prime}}{O A B}=\frac{m^{\prime} n^{\prime}}{m n},
$$


und daher

$$
O B C: O C A: O A B=O^{\prime} B^{\prime} C^{\prime}: O^{\prime} C^{\prime} A^{\prime}: O^{\prime} A^{\prime} B^{\prime} .
$$

Der Punkt $O$ wird der Schwerpunkt der Punkte $A, B, C$, wenn man an diesen den Dreiecken $O B C, O C A, O A B$ proportionale Gewichte anbringt. Ebenso wird $O^{\prime}$ der Schwerpunkt der Punkte $A^{\prime}, B^{\prime}, C^{\prime}$, wenn man an ihnen den Dreiecken $O^{\prime} B^{\prime} C^{\prime}, O^{\prime} C^{\prime} A^{\prime}, O^{\prime} A^{\prime} B^{\prime}$ proportionale Gewichte anbringt. Die Gewichte, welche man an $A, B, C$ anzubringen hat, um den Punkt $O$ zum Schwerpunkt zu erhalten, können daher den Gewichten gleich gesetzt werden, welche man an $A^{\prime}, B^{\prime}, C^{\prime}$ anzubringen hat, um den Punkt $O^{\prime}$ zu ihrem Schwerpunkt zu erhalten.

Die an $A, B, C$ angebrachten Gewichte mögen $\alpha, \beta, \gamma$ heissen, und ihre Summe $\mu$, so wird die Entfernung eines beliebigen Punktes $P$ vom Schwerpunkt $O$ durch die bekannte Formel:

$$
\mu . P O^{2}=\alpha \cdot P A^{2}+\beta \cdot P B^{2}+\gamma \cdot P C^{2}-\frac{1}{\mu}\left\{\beta \gamma \cdot B C^{2}+\gamma \alpha \cdot C A^{2}+\alpha \beta . A B^{2}\right\}
$$

gegeben.

Ebenso wird die Entfernung eines Punktes $P^{\prime}$ von $O^{\prime}$ durch die Formel:

$$
\mu . P^{\prime} O^{\prime 2}=\alpha . P^{\prime} A^{\prime 2}+\beta . P^{\prime} B^{\prime 2}+\gamma \cdot P^{\prime} C^{\prime 2}-\frac{1}{\mu}\left\{\beta \gamma . B^{\prime} C^{\prime 2}+\gamma \alpha . C^{\prime} A^{\prime 2}+\alpha \beta . A^{\prime} B^{\prime 2}\right\}
$$

gegeben. Sind $\boldsymbol{P}$ und $\boldsymbol{P}^{\prime}$ zwei conjugirte Punkte, und ist

so hat man auch

$$
p^{2}=m^{2}-m^{\prime 2}=n^{2}-n^{\prime 2}
$$

$$
p^{2}=P O^{2}-P^{\prime} O^{\prime 2}
$$

und daher, wenn man die angegebenen Werthe von $\mu P O^{2}$ und $\mu P^{\prime} O^{\prime 2}$ substituirt und der Kürze halber

setzt,

$$
B C^{2}-B^{\prime} C^{\prime 2}=u, \quad C A^{2}-C^{\prime} A^{\prime 2}=v, \quad A B^{2}-A^{\prime} B^{\prime 2}=w
$$

$\mu p^{2}=\alpha\left(P A^{2}-P^{\prime} A^{\prime 2}\right)+\beta\left(P B^{2}-P^{\prime} B^{\prime 2}\right)+\gamma\left(P C^{2}-P^{\prime} C^{\prime 2}\right)-\frac{1}{\mu}\{\beta \gamma . u+\gamma \alpha . v+\alpha \beta . w\}$.

Lässt man $P$ nach und nach mit $A, B, C$ und gleichzeitig $P^{\prime}$ mit $A^{\prime}, B^{\prime}, C^{\prime}$ zusammenfallen, so giebt diese Formel die Gleichungen:

$$
\mu p^{2}+\frac{1}{\mu}(\beta \gamma . u+\gamma \alpha \cdot v+\alpha \beta \cdot w)=\beta w+\gamma v=\gamma u+\alpha w=\alpha v+\beta u \text {. }
$$

Aus diesen Gleichungen folgt, dass die Gewichte $\alpha, \beta, \gamma$ den Grössen $u(v+w-u), v(w+u-v), w(u+v-v)$ proportional sind. Setzt man sie diesen Grössen gleich, so folgen aus den Werthen

$$
u(v+v-u)=\alpha, \quad v(w+u-v)=\beta, \quad w(u+v-w)=\gamma
$$


die Gleichungen,

ferner

$$
\begin{aligned}
& \mu=2(v w+w u+u v)-u^{2}-v^{2}-v^{2} \\
& \mu p^{2}+\frac{1}{\mu}(\beta \gamma . u+\gamma \alpha . v+\alpha \beta . v)=2 u v w
\end{aligned}
$$

$\beta \gamma \cdot u+\gamma \alpha \cdot v+\alpha \beta . w=u v w\left[u^{2}+v^{2}+w^{2}-(v-v)^{2}-(w-u)^{2}-(u-v)^{2}\right]=u v v \mu$ und hieraus

Wir haben daher den Satz:

$$
p^{2}=\frac{u v w}{\mu} .
$$

Wenn zwei den beliebig gegebenen Dreiecken $A B C$ und $A^{\prime} B^{\prime} C^{\prime}$ umschriebene Kegelschnitte gleiche Excentricität haben, und in ihnen $A$ und $A^{\prime}$, $B$ und $B^{\prime}, C$ und $C^{\prime}$ conjugirte Punkte sind, so erhält man ihre Mittelpunkte $O$ und $O^{\prime}$ als die Schwerpunkte von $A, B, C$ und von $A^{\prime}, B^{\prime}, C^{\prime}$, wenn man in diesen drei Punkten respective die Gewichte

anbringt, wo

$$
u(v+v-u), \quad v(v+u-v), \quad w(u+v-w)
$$

$$
u=B C^{2}-B^{\prime} C^{\prime 2}, \quad v=C A^{2}-C^{\prime} A^{\prime 2}, \quad v=A B^{2}-A^{\prime} B^{\prime 2}
$$

ist, und es wird die Differenz der Quadrate der halben grossen oder der halben kleinen Achsen der Kegelschnitte:

$$
\frac{u v w}{2(v w+w u+u v)-u^{2}-v^{2}-v^{2}} .
$$

Nimmt man in jedem der beiden Kegelschnitte die beiden Hauptachsen zu Coordinatenachsen und setzt die Coordinaten von $B$ und $C$

$$
m \cos \eta, \quad n \sin \eta \quad \text { und } m \cos \vartheta, \quad n \sin \vartheta \text {, }
$$

so werden die Coordinaten von $B^{\prime}$ und $C^{\prime}$

$$
m^{\prime} \cos \eta, \quad n^{\prime} \sin \eta \quad \text { und } \quad m^{\prime} \cos \vartheta, \quad n^{\prime} \sin \vartheta
$$

hieraus folgt, da $m^{2}-m^{\prime 2}=n^{2}-n^{\prime 2}=p^{2}$,

$$
\begin{aligned}
u=B C^{2}-B^{\prime} C^{\prime 2} & =p^{2}\left((\cos \eta-\cos \vartheta)^{2}+(\sin \eta-\sin \vartheta)^{2}\right) \\
& =2 p^{2}(1-\cos (\eta-\vartheta))=4 p^{2} \sin ^{2} \frac{\eta-\vartheta}{2} .
\end{aligned}
$$

Nennt man $\triangle$ den Inhalt des gegebenen Dreieckes $A B C$, so wird der Inhalt des Dreieckes $O B C$ gleich $\frac{\alpha \Delta}{\mu}$. Andrerseits wird der Inhalt desselben Dreiecks

und daher

$$
\pm \frac{1}{2} m n \sin (\eta-9)
$$

$$
\frac{\alpha^{2} \Delta^{2}}{m^{2} n^{2} \mu^{2}}=\frac{1}{4} \sin ^{2}(\eta-\vartheta)
$$


dividirt man diese Gleichung durch

so erhält man:

$$
\frac{u}{4 p^{2}}=\sin ^{2} \frac{\eta-\vartheta}{2}
$$

und daher

$$
\frac{4 p^{2} \Delta \alpha^{2}}{m^{2} n^{2} \mu^{2} u}=\cos ^{2} \frac{\eta-\vartheta}{2}
$$

$$
\frac{4 p^{2} \Delta^{2} \alpha^{2}}{m^{2} n^{2} \mu^{2} u}=1-\frac{u}{4 p^{2}} .
$$

Quadrirt man den Werth von $\alpha=u(v+w-u)$ und vergleicht den für $\mu$ gefundenen Werth, so ergiebt sich

$$
\alpha^{2}=u^{2}(4 v w-\mu)=4 u^{2} v w\left(1-\frac{u}{4 p^{2}}\right) .
$$

Die vorstehende Gleichung verwandelt sich daher nach Division durch $1-\frac{u}{4 p^{2}}$ in die folgende:

$$
\left.\frac{16 p^{2} \Delta^{2} u v w}{m^{2} n^{2} \mu^{2}}=\frac{16 \Delta^{2} u^{2} v^{2} v^{2}}{m^{2} n^{2} \mu^{3}}=1^{*}\right) \text {. }
$$

Nennt man $A^{\prime}$ den Inhalt des Dreieckes $A^{\prime} B^{\prime} C^{\prime}$, so erhält man eine ganz analoge Gleichung. Aus den beiden so gefundenen Gleichungen

$$
\begin{aligned}
m^{2} n^{2} & =\frac{16 u^{2} v^{2} w^{2}}{\mu^{3}} \Delta^{2}=\frac{16 p^{4}}{\mu} \Delta^{2}, \\
m^{\prime 2} n^{\prime 2} & =\frac{16 u^{2} v^{2} w^{2}}{\mu^{3}} \Delta^{\prime 2}=\frac{16 p^{4}}{\mu} \Delta^{\prime 2}
\end{aligned}
$$

folgt, wenn man abzieht und durch $p^{2}$ dividirt:

und daher

$$
m^{2}+n^{2}-p^{2}=\frac{16 p^{2}}{\mu}\left(\Delta^{2}-\Delta^{2}\right)
$$

$$
m^{2}+n^{2}=\frac{16 p^{2}}{\mu}\left(\Delta^{2}-\Delta^{\prime 2}+\frac{\mu}{16}\right) .
$$

Hieraus folgt die vierte Potenz der halben Excentricität beider Kegelschnitte:

oder

$$
\left(m^{2}-n^{2}\right)^{2}=\frac{256 p^{4}}{\mu^{2}}\left(\Delta^{2}-\Delta^{\prime 2}\right)^{2}-\frac{32 p^{4}}{\mu}\left(\Delta^{2}+\Delta^{\prime 2}\right)+p^{4}
$$

$$
\begin{aligned}
\frac{\mu^{2}\left(m^{2}-n^{2}\right)^{2}}{p^{4}} & =\left(16\left(\Delta^{2}+\Delta^{\prime 2}\right)-\mu\right)^{2}-1024 \Delta^{2} \Delta^{\prime 2} \\
& =\left(16\left(A+\Delta^{\prime}\right)^{2}-\mu\right)\left(16\left(\Delta-\Delta^{\prime}\right)^{2}-\mu\right) .
\end{aligned}
$$

*) Die Herleitung dieser Gleichung wäre nur dann unstatthaft, wenn $u=v=v=4 p^{2}$. Wenn $u=v=v$, wird aber $\mu=3 u^{2}, p^{2}=\frac{1}{3} u$, es müsste also $u=v=v=0$ sein, oder beide Dreiecke congruent werden, in welchem Falle die ganze Aufgabe unstatthaft ist. 
Man erhält aber auch einen merkwürdigen Ausdruck, wenn man die vorstehende Grösse durch die unmittelbar gegebenen Seiten der Dreiecke $A B C$ und $A^{\prime} B^{\prime} C^{\prime}$ darstellt. Es sei

so wird

$$
\begin{aligned}
& B C=a, \quad C A=b, \quad A B=c, \\
& B^{\prime} C^{\prime}=a^{\prime}, \quad C^{\prime} A^{\prime}=b^{\prime}, \quad A^{\prime} B^{\prime}=c^{\prime},
\end{aligned}
$$

und daher

$$
\begin{gathered}
16 \Delta^{2}=2\left(b^{2} c^{2}+c^{2} a^{2}+a^{2} b^{2}\right)-a^{4}-b^{4}-c^{4}, \\
16 \Delta^{\prime 2}=2\left(b^{\prime 2} c^{\prime 2}+c^{\prime 2} a^{\prime 2}+a^{\prime 2} b^{\prime 2}\right)-a^{\prime 4}-b^{\prime 4}-c^{\prime 4}, \\
\mu=\left\{\begin{array}{l}
2\left\{\left(b^{2}-b^{\prime 2}\right)\left(c^{2}-c^{\prime 2}\right)+\left(c^{2}-c^{\prime 2}\right)\left(a^{2}-a^{\prime 2}\right)+\left(a^{2}-a^{\prime 2}\right)\left(b^{2}-b^{\prime 2}\right)\right\} \\
-\left(a^{2}-a^{\prime 2}\right)^{2}-\left(b^{2}-b^{\prime 2}\right)^{2}-\left(c^{2}-c^{\prime 2}\right)^{2}
\end{array}\right.
\end{gathered}
$$

$$
16\left(\Delta^{2}+\Delta^{\prime 2}-\frac{\mu}{16}\right)=2\left\{a^{\prime 2}\left(b^{2}+c^{2}-a^{2}\right)+b^{\prime 2}\left(c^{2}+a^{2}-b^{2}\right)+c^{\prime 2}\left(a^{2}+b^{2}-c^{2}\right)\right\} .
$$

Bemerkt man die Formeln

$$
\begin{gathered}
\left(b^{2}+c^{2}-a^{2}\right)^{2}=4 b^{2} c^{2}-16 A^{2}, \\
\left(c^{2}+a^{2}-b^{2}\right)\left(a^{2}+b^{2}-c^{2}\right)=a^{4}-b^{4}-c^{4}+2 b^{2} c^{2}=16 A^{2}-2 a^{2}\left(b^{2}+c^{2}-a^{2}\right)
\end{gathered}
$$

und die ähnlichen, so erhält man

$$
\begin{gathered}
256\left(\Delta^{2}+\Delta^{\prime 2}-\frac{\mu}{16}\right)^{2}-1024 \Delta^{2} \Delta^{\prime 2} \\
=\left\{\begin{array}{l}
16\left(a^{\prime \prime} b^{2} c^{2}+b^{\prime 4} c^{2} a^{2}+c^{\prime 4} a^{2} b^{2}\right) \\
-16\left\{b^{\prime 2} c^{\prime 2} a^{2}\left(b^{2}+c^{2}-a^{2}\right)+c^{\prime 2} a^{\prime 2} b^{2}\left(c^{2}+a^{2}-b^{2}\right)+a^{\prime 2} b^{\prime 2} c^{2}\left(a^{2}+b^{2}-c^{2}\right)\right\} .
\end{array}\right.
\end{gathered}
$$

Setzt man der Kürze halber

$$
b^{2} c^{\prime 2}-c^{2} b^{\prime 2}=\mathfrak{A}, \quad c^{2} a^{\prime 2}-a^{2} c^{\prime 2}=\mathfrak{B}, \quad a^{2} b^{\prime 2}-b^{2} a^{\prime 2}=\mathfrak{E},
$$

so kann man dem vorstehenden Ausdruck folgende elegante Form geben

$$
\mu^{4} \frac{\left(m^{2}-n^{2}\right)^{2}}{u^{2} v^{2}} \frac{w^{2}}{w^{2}}=-16(\mathfrak{B} \mathfrak{C}+\mathfrak{Q}+\mathfrak{A} \mathfrak{B}) \text {. }
$$

Aus der ganzen Natur der Aufgabe geht hervor, dass die vorstehende Grösse positiv ist. Man sieht es aber nicht so leicht aus der Art, wie dieselbe zusammengesetzt ist, daher ich dies kurz beweisen will.

$$
\text { Man setze } \frac{b^{2}}{b^{\prime 2}}-\frac{c^{2}}{c^{\prime 2}}=d, \frac{c^{2}}{c^{\prime 2}}-\frac{a^{2}}{a^{\prime 2}}=d^{\prime}, \frac{a^{2}}{a^{\prime 2}}-\frac{b^{2}}{b^{\prime 2}}=d^{\prime \prime},
$$

so hat man $d+d^{\prime}+d^{\prime \prime}=0$, ferner

$$
\mathfrak{A}=b^{\prime 2} c^{\prime 2} d, \quad \mathfrak{B}=c^{\prime 2} a^{\prime 2} d^{\prime}, \quad \mathfrak{S}=a^{\prime 2} b^{\prime 2} d^{\prime \prime}
$$

Journal für Mathematik Bd. LXXIII. Heft 2. 
und daher:

$$
\mathfrak{B C}+\mathfrak{E A}+\mathfrak{A} \mathfrak{B}=a^{\prime 2} b^{\prime 2} c^{\prime 2}\left(a^{\prime 2} d^{\prime} d^{\prime \prime}+b^{\prime 2} d^{\prime \prime} d+c^{\prime 2} d d^{\prime}\right) .
$$

Es ist daher zu beweisen, dass der in Klammern eingeschlossene Ausdruck immer negativ ist. Da $d+d^{\prime}+d^{\prime \prime}=0$, so müssen zwei der Grössen dasselbe Zeichen haben, während die dritte Grösse das entgegengesetzte Zeichen hat. Haben z. B. $d^{\prime}$ und $d^{\prime \prime}$ dasselbe Zeichen, so sieht man, dass die in Klammern eingeschlossene Grösse negativ wird, wenn man ihr die Form giebt:

$$
d^{\prime} d^{\prime \prime}\left(a^{\prime 2}-b^{\prime 2}-c^{\prime 2}\right)-b^{\prime 2} d^{\prime \prime 2}-c^{\prime 2} d^{\prime 2}=-d^{\prime} d^{\prime \prime}\left(\left(b^{\prime}+c^{\prime}\right)^{2}-a^{\prime 2}\right)-\left(b^{\prime} d^{\prime \prime}-c^{\prime} d^{\prime}\right)^{2},
$$

da immer von den drei Seiten des Dreiecks $A^{\prime} B^{\prime} C^{\prime}$ die Summe von zweien $b^{\prime}+c^{\prime}$ grösser als die dritte $a^{\prime}$, also $\left(b^{\prime}+c^{\prime}\right)^{2}-a^{\prime 2}$ positiv ist.

Wenn $m^{2}-n^{2}=m^{\prime 2}-n^{\prime 2}=0$, so muss man $\mathfrak{A}=\mathfrak{B}=\mathfrak{C}$ haben, oder: sollen die Kegelschnitte Kreise werden, so müssen die Dreiecke $A B C$ und $A^{\prime} B^{\prime} C^{\prime}$ einander ähnlich sein.

Aus den Gleichungen:

folgt

$$
\begin{aligned}
16\left(\Delta^{2}+\Delta^{\prime 2}-\frac{\mu}{16}\right) & =2\left\{a^{\prime 2}\left(b^{2}+c^{2}-a^{2}\right)+b^{\prime 2}\left(c^{2}+a^{2}-b^{2}\right)+c^{\prime 2}\left(a^{2}+b^{2}-c^{2}\right)\right\} \\
& =2\left\{a^{2}\left(b^{\prime 2}+c^{\prime 2}-a^{\prime 2}\right)+b^{2}\left(c^{\prime 2}+a^{\prime 2}-b^{\prime 2}\right)+c^{2}\left(a^{\prime 2}+b^{\prime 2}-c^{\prime 2}\right)\right\} . \\
16 \Delta^{2} & =a^{2}\left(b^{2}+c^{2}-a^{2}\right)+b^{2}\left(c^{2}+a^{2}-b^{2}\right)+c^{2}\left(a^{2}+b^{2}-c^{2}\right) \\
16 A^{\prime 2} & =a^{\prime 2}\left(b^{\prime 2}+c^{\prime 2}-a^{\prime 2}\right)+b^{\prime 2}\left(c^{\prime 2}+a^{\prime 2}-b^{\prime 2}\right)+c^{\prime 2}\left(a^{\prime 2}+b^{\prime 2}-c^{\prime 2}\right)
\end{aligned}
$$

$$
\begin{aligned}
\mu \frac{\left(m^{2}+n^{2}\right)}{2 p^{2}} & =8\left(\Delta^{2}-\Delta^{\prime 2}+\frac{\mu}{16}\right) \\
& =u\left(b^{2}+c^{2}-a^{2}\right)+v\left(c^{2}+a^{2}-b^{2}\right)+w\left(a^{2}+b^{2}-c^{2}\right), \\
\mu \frac{\left(m^{\prime 2}+n^{\prime 2}\right)}{2 p^{2}} & =8\left(\Delta^{2}-\Delta^{\prime 2}-\frac{\mu}{16}\right) \\
& =u\left(b^{\prime 2}+c^{\prime 2}-a^{\prime 2}\right)+v\left(c^{\prime 2}+a^{\prime 2}-b^{\prime 2}\right)+w\left(a^{\prime 2}+b^{\prime 2}-c^{\prime 2}\right) .
\end{aligned}
$$

Wenn $\mu$ und $u, v, w$ positiv sind, müssen diese beiden Ausdrücke ebenfalls positiv werden, was von dem ersten zu beweisen hinreicht. Wenn die drei Grössen $b^{2}+c^{2}-a^{2}, c^{2}+a^{2}-b^{2}, a^{2}+b^{2}-c^{2}$ positiv sind, erhellt dies von selbst. Es kann aber eine der drei Grössen, z. B. $b^{2}+c^{2}-a^{2}$, negativ werden. In diesem Falle stelle man den Ausdruck so dar:

$$
\begin{aligned}
& u\left(b^{2}+c^{2}-a^{2}\right)+v\left(c^{2}+a^{2}-b^{2}\right)+w\left(a^{2}+b^{2}-c^{2}\right) \\
= & 2\left(v c^{2}+w b^{2}\right)-\left(a^{2}-b^{2}-c^{2}\right)(u-v-w),
\end{aligned}
$$

wodurch man sieht, dass er positiv ist, wenn $u<v+w$. Ist $u>v+v$, so hat man doch $u-v-w<2 \sqrt{ } v w$, denn damit

$$
\mu=(\sqrt{ } u+\sqrt{ } v+\sqrt{ } w)(\sqrt{ } v+\sqrt{ } w-\sqrt{ } u)(\sqrt{ } w+\sqrt{ } u-\sqrt{ } v)(\sqrt{ } u+\sqrt{ } v-\sqrt{ } w)
$$


positiv sei, muss von den drei Grössen $\sqrt{ } u, \sqrt{ } v, \sqrt{ } w$ eine immer kleiner als die Summe der beiden andern sein. Ebenso hat man, wenn $a^{2}>b^{2}+c^{2}$, doch $a^{2}-b^{2}-c^{2}<2 b c$ und daher $\left(a^{2}-b^{2}-c^{2}\right)(u-v-w)<4 b c \downarrow v w$. Da nun $2\left(v c^{2}+w b^{2}\right)>4 b c \downarrow v w$, so wird der vorgelegte Ausdruck immer positiv. Man leitet hieraus leicht die Folgerung ab, dass die Grössen $m^{2}$ und $n^{2}$ nie zugleich negativ werden können.

Wenn $\mu$ positiv ist, so müssen die Seiten des Dreiecks $A B C$ alle grösser oder alle kleiner sein als die entsprechenden Seiten des Dreiecks $A^{\prime} B^{\prime} C^{\prime}$. Ferner müssen die Differenzen der Quadrate der entsprechenden Seiten beider Dreiecke, oder die Grössen $u, v$, $w$, so beschaffen sein, dass man $\sqrt{ } \pm u, \sqrt{ } \pm v, \sqrt{ } \pm w$ zu Seiten eines Dreiecks nehmen könne, welches ich $A^{\prime \prime} B^{\prime \prime} C^{\prime \prime}$ nennen und dessen Inhalt ich mit $\Delta^{\prime \prime}$ bezeichnen will.' Man kann mit Hilfe dieses Dreiecks mehrere der angegebenen Formeln elegant construiren. Zuerst wird

$$
\mu=16 \Delta^{\prime \prime} A^{\prime \prime},
$$

ferner, da $p=\frac{\sqrt{\text { uvw }}}{4 \Delta^{\prime \prime}}$, so wird $p$ gleich dem Radius des dem Dreieck $A^{\prime \prime} B^{\prime \prime} C^{\prime \prime}$ umschriebenen Kreises. Die Gewichte $\alpha, \beta, \gamma$ verhalten sich ferner, wie die Sinus der doppelten Winkel des Dreiecks $A^{\prime \prime} B^{\prime \prime} C^{\prime \prime}$, oder verhalten sich wie die Gewichte an den Ecken des Dreiecks $A^{\prime \prime} B^{\prime \prime} C^{\prime \prime}$, deren Schwerpunkt der Mittelpunkt des diesem umschriebenen Kreises ist.

Man hat ferner

$$
\begin{aligned}
& \frac{p^{2}}{\Delta^{\prime \prime}}=\frac{m n}{\Delta}=\frac{m^{\prime} n^{\prime}}{\Delta^{\prime}}, \\
& \left.\frac{\Delta^{\prime \prime 4}\left(m^{2}-n^{2}\right)^{2}}{p^{4}}=\dot{(} \Delta+\Delta^{\prime}+\Delta^{\prime \prime}\right)\left(\Delta+\Delta^{\prime}-\Delta^{\prime \prime}\right)\left(\Delta-\Delta^{\prime}+\Delta^{\prime \prime}\right)\left(\Delta-\Delta^{\prime}-\Delta^{\prime \prime}\right) \text {. }
\end{aligned}
$$

Diese letzte Formel lehrt, dass unter den Dreiecken $\Delta, \Delta^{\prime}, \Delta^{\prime \prime}$ eins grösser als die Summe der beiden andern sein muss. Die Combination beider Formeln führt auf die Gleichung:

$$
p^{4}\left(m^{2}-n^{2}\right)^{2}=\left(m n+m^{\prime} n^{\prime}+p p\right)\left(m n+m^{\prime} n^{\prime}-p p\right)\left(m n-m^{\prime} n^{\prime}+p p\right)\left(m n-m^{\prime} n^{\prime}-p p\right),
$$

welche durch die Substitution der Werthe $m^{\prime 2}=m^{2}-p^{2}, n^{\prime 2}=n^{2}-p^{2}$ identisch wird.

Aus den Gleichungen

$$
m^{2} n^{2}=\frac{16 u^{2} v^{2} w^{2} \Delta^{2}}{\mu^{3}}, \quad m^{\prime 2} n^{\prime 2}=\frac{16 u^{2} v^{2} w^{2} \Delta^{\prime 2}}{\mu^{3}}
$$

folgt, dass die beiden Kegelschnitte Ellipsen oder Hyperbeln sind, jenachdem $\mu$ positiv oder negativ ist. Denn es tritt das eine oder das andere ein, 
jenachdem $m^{2} n^{2}$ positiv oder negativ ist. Die vorstehenden Constructionen gelten daher für den Fall, dass die Kegelschnitte Ellipsen sind, und umgekehrt: es werden die Kegelschnitte Ellipsen, wenn das Hilfsdreieck $A^{\prime \prime} B^{\prime \prime} C^{\prime \prime}$ reell wird.

Ich will das im Vorstehenden für die Beschaffenheit der Kegelschnitte gefundene Kennzeichen noch auf eine andere Art beweisen, und zwar vermittelst eines schönen Satzes, welchen Steiner im XCIX ${ }^{\text {ten }}$ Bande des in Rom erscheinenden Giornale Arcadico bekannt gemacht hat ${ }^{*}$ ). Wenn man nämlich um ein Dreieck $A B C$ einen Kegelschnitt beschreibt, dessen Mittelpunkt ein gegebener Punkt $O$ ist, und das Dreieck $M M^{\prime} M^{\prime \prime}$, dessen Ecken die Mitten der Seiten des Dreiecks $A B C$ sind, construirt, so wird zufolge dieses Satzes der Kegelschnitt eine Ellipse, wenn $O$ im Innern des Dreiecks $M M^{\prime} M^{\prime \prime}$ liegt oder in einem der drei unendlichen Räume, die an einer der Ecken des Dreiecks $M M^{\prime} M^{\prime \prime}$ von den Verlängerungen seiner zwei dort zusammenstossenden Seiten eingeschlossen werden; wenn dagegen der Mittelpunkt $O$ in einem der drei unendlichen Räume liegt, welche von einer der Seiten des Dreiecks und den Verlängerungen der beiden andern umschlossen werden, so wird der Kegelschnitt eine Hyperbel. Ist $O$ als Schwerpunkt der drei Punkte $A, B, C$ gegeben, wenn man in ihnen die Gewichte $\alpha, \beta, \gamma$ anbringt, und sind $M, M^{\prime}, M^{\prime \prime}$ die Mitten der Seiten $B C, C A, A B$, so wird $O$ auch der Schwerpunkt der Punkte $M, M^{\prime}, M^{\prime \prime}$, wenn man in ihnen die Gewichte

$$
\alpha^{\prime}=\beta+\gamma-\alpha, \quad \beta^{\prime}=\gamma+\alpha-\beta, \quad \gamma^{\prime}=\alpha+\beta-\gamma
$$

anbringt. Haben diese drei Gewichte $\alpha^{\prime}, \beta^{\prime}, \gamma^{\prime}$ dasselbe Zeichen, so fällt ihr Schwerpunkt $O$ in das Innere des Dreiecks $M M^{\prime} M^{\prime \prime}$. Haben zwei Gewichte, z. B. $\beta^{\prime}$ und $\gamma^{\prime}$, dasselbe Zeichen, aber das dritte Gewicht $\alpha^{\prime}$ das entgegengesetzte, so fällt $O$ entweder in den bei $M$ oder in den über $M^{\prime} M^{\prime \prime}$ gebildeten unendlichen Raum, jenachdem $\alpha^{\prime}+\beta^{\prime}+\gamma^{\prime}$ dasselbe Zeichen wie $\alpha^{\prime}$ oder wie $\beta^{\prime}$ und $\gamma^{\prime}$ hat. Man kann daher sagen, dass der Kegelschnitt eine Ellipse oder Hyperbel wird, jenachdem $\alpha^{\prime}+\beta^{\prime}+\gamma^{\prime}$ dasselbe oder das entgegengesetzte Zeichen wie $\alpha^{\prime} \beta^{\prime} \gamma^{\prime}$ hat, oder man hat den Satz:

Wenn ein Kegelschnitt durch drei mit den Gewichten $\alpha, \beta, \gamma$ behaftete Punkte geht und ihren Schwerpunkt zum. Mittelpunkt hat, so wird der Kegelschnitt eine Ellipse oder Hyperbel, jenachdem die Grösse

positiv oder negativ ist.

$$
(\alpha+\beta+\gamma)(\beta+\gamma-\alpha)(\gamma+\alpha-\beta)(\alpha+\beta-\gamma)
$$

*) Wieder abgedruckt im $30^{\text {ten }}$ Bande dieses Journals p. 97. 
Für die oben (S. 191) angegebenen Werthe von $\alpha, \beta, \gamma$ wird

$$
\begin{aligned}
& \alpha^{\prime}=\beta+\gamma-\alpha=(u+v-w)(u-v+w)=\frac{\beta \gamma}{v w}, \\
& \beta^{\prime}=\gamma+\alpha-\beta=(v+v-u)(v-w+u)=\frac{\gamma \alpha}{w u}, \\
& \gamma^{\prime}=\alpha+\beta-\gamma=(w+u-v)(w-u+v)=\frac{\alpha \beta}{u v} .
\end{aligned}
$$

Da das Product dieser drei Grössen immer positiv ist, so hat das Product der vier Grössen in dem eben bewiesenen Satze dasselbe Zeichen wie $\alpha+\beta+\gamma=\mu$, und es wird daher der Kegelschnitt eine Ellipse oder Hyperbel, jenachdem $\mu$ positiv oder negativ ist. Man kann noch bemerken, dass der Punkt $O$ nie in die Seiten des Dreiecks $M M^{\prime} M^{\prime \prime}$ oder in ihre Verlängerungen fallen kann, wenn er nicht einer der Punkte $M, M^{\prime}, M^{\prime \prime}$ selber wird; denn es müsste in jenem Falle eins der Gewichte in den Punkten $M, M^{\prime}, M^{\prime \prime}$ zufolge der Beschaffenheit der Werthe dieser Gewichte verschwinden, welches aber nicht möglich ist, ohne dass noch ein anderes Gewicht verschwindet, in welchem Falle der Schwerpunkt mit dem dritten Punkte zusammenfällt. Wird z. B. $v+w-u=0$, so verschwinden $\beta^{\prime}, \gamma^{\prime}, \alpha$; es wird ferner $\beta=\gamma=2 v v, \mu=4 v w$,

$$
p^{2}=\frac{1}{4} u, \quad m^{2}+n^{2}=u\left(\frac{c^{2}}{w}+\frac{b^{2}}{v}\right), \quad m^{2} n^{2}=\frac{u^{2} \Delta}{4 v w} .
$$

Die Kegelschnitte bleiben in diesem Falle bei unserer Aufgabe vollkommen bestimmt, obgleich im Allgemeinen die Aufgabe, einen Kegelschnitt mit gegebenem Mittelpunkt einem gegebenen Dreieck zu umschreiben, unbestimmt wird, wenn der gegebene Mittelpunkt in die Mitte einer der Seiten des Dreiecks fällt. Der hier betrachtete Fall tritt ein, wenn das Hilfsdreieck $A^{\prime \prime} B^{\prime \prime} C^{\prime \prime}$ rechtwinklig ist.

Setzt man wieder, wie oben, die Coordinaten der Punkte $B$ und $C$ gleich $m \cos \eta, \quad n \sin \eta \quad$ und $m \cos \vartheta, \quad n \sin \vartheta$

so wird die Tangente des Winkels, welchen $B C$ mit der grossen Achse bildet,

$$
\frac{n(\sin \eta-\sin \vartheta)}{m(\cos \eta-\cos \vartheta)}=\frac{n}{m} \frac{\sin \left(\frac{\eta+\vartheta}{2}+90^{\circ}\right)}{\cos \left(\frac{\eta+\vartheta}{2}+90^{\circ}\right)}
$$

- Der Punkt $D$, in welchem der mit $B C$ parallele Durchmesser den Kegelschnitt. trifft, hat daher die Coordinaten:

$$
m \cos \left(\frac{\eta+\vartheta}{2}+90^{\prime \prime}\right), \quad n \sin \left(\frac{\eta+\vartheta}{2}+90^{\prime \prime}\right)
$$


und es wird

$$
O D^{2}=m^{2} \sin ^{2} \frac{\eta+\vartheta}{2}+n^{2} \cos ^{2} \frac{\eta+\vartheta}{2}=\frac{B C^{2}}{\sin ^{2} \frac{\eta-\vartheta}{2}}=\frac{a^{2}}{\sin ^{2} \frac{\eta-\vartheta}{2}} .
$$

Substituirt man hierin den Werth für $\sin ^{2} \frac{\eta-\vartheta}{2}=\frac{u}{4 p^{2}}=\frac{\mu}{4 v w}$ und bildet die beiden andern ähnlichen Formeln, so findet man für die Quadrate der den Seiten $B C, C A, A B$ parallelen halben Durchmesser die Werthe:

$$
\frac{v w a^{2}}{\mu}, \frac{w u b^{2}}{\mu}, \frac{u v c^{2}}{\mu} \text {. }
$$

Ebenso werden die Quadrate der den Seiten $B^{\prime} C^{\prime}, C^{\prime} A^{\prime}, A^{\prime} B^{\prime}$ parallelen halben Durchmesser

$$
\frac{v w a^{\prime 2}}{\mu}, \frac{v u b^{\prime 2}}{\mu}, \frac{u v c^{\prime 2}}{\mu},
$$

und die Differenz je zweier entsprechenden Grössen wird

$$
\frac{u v w}{\mu}=p^{2} \text {. - }
$$

Die beiden Kegelschnitte sind im Allgemeinen schon durch drei ihrer Punkte $A, B, C, A^{\prime}, B^{\prime}, C^{\prime}$, und durch ihre Mittelpunkte $O$ und $O^{\prime}$ vollkommen bestimmt. Legt man ihre Mittelpunkte und grossen Achsen in einander, so erhält man diejenige Lage, in welcher ihre beiden Brennpunkte zusammenfallen, auf welche sich unsere ursprüngliche Betrachtung bezog. In dieser Lage wird nach dem Ivoryschen Theorem

$$
B C^{\prime}=C B^{\prime}, \quad C A^{\prime}=A C^{\prime}, \quad A B^{\prime}=B A^{\prime} .
$$

Es ist also durch die angestellten Untersuchungen auch die Aufgabe gelöst:

Zwei beliebige Dreiecke $A B C$ und $A^{\prime} B^{\prime} C^{\prime}$ in solche gegenseitige Lage zu bringen, dass $B C^{\prime}=C B^{\prime}, C A^{\prime}=A C^{\prime}, A B^{\prime}=B A^{\prime}$ wird.

Setzt man nämlich, wie oben,

$$
B C^{2}-B^{\prime} C^{\prime 2}=u, \quad C A^{2}-C^{\prime} A^{\prime 2}=v, \quad A B^{2}-A^{\prime} B^{\prime 2}=w,
$$

und bringt in $A, B, C$ und ebenso in $A^{\prime}, B^{\prime}, C^{\prime}$ die Gewichte

$$
u(v+w-u), \quad v(w+u-v), \quad w(u+v-w)
$$

an, deren Schwerpunkte $O$ und $O^{\prime}$ seien, so sucht man die grossen Achsen der beiden Kegelschnitte, die den Dreiecken $A B C, A^{\prime} B^{\prime} C^{\prime}$ umschrieben sind und respective $O$ und $O^{\prime}$ zu Mittelpunkten haben. Denkt man sich die Punkte 
$O$ und $O^{\prime}$ und diese grossen Achsen in den Dreiecken fest und legt sie auf einander, so erbalten die Dreiecke die verlangte Lage. -

Eine besondere Betrachtung macht der Fall nothwendig, wenn zwei correspondirende Seiten der beiden Basisdreiecke einander gleich sind. Denn die Analysis, durch welche oben die Gewichte $\alpha, \beta, \gamma$ und der Werth von $p^{2}$ gefunden wurden, setzt voraus, dass keine der Grössen $u, v, w$ verschwinde. Es sei z. B. $B C=B^{\prime} C^{\prime}$, also $u=0$, so geben die gefundenen Formeln

$$
\begin{gathered}
u=0, \quad \alpha=0, \quad p^{2}=0, \\
\beta=-v(v-w), \quad \gamma=w(v-w), \quad \mu=-(\dot{v}-w)^{2} .
\end{gathered}
$$

Die Verhältnisse von $m^{2}, n^{2}, p^{2}$ bleiben endlich. Die Punkte $O$ und $O^{\prime}$ kommen in die Linien $B C$ und $B^{\prime} C^{\prime}$ selbst zu liegen. Lässt man $B$ mit $B^{\prime}, C$ mit $C^{\prime}$ zusammenfallen, so fällt auch $O$ in $O^{\prime}$. Man findet dann diesen Punkt durch eine leichte Construction, indem man auf $A A^{\prime}$ in der Mitte von $\boldsymbol{A} \boldsymbol{A}^{\prime}$ ein Perpendikel errichtet, dessen Durchschnitt mit der Linie $B^{\prime} C^{\prime}$ den Punkt $O$ giebt. Die beiden Kegelschnitte verwandeln sich in die Systeme zweier geraden Linien, von denen die eine $B C$, die beiden anderen $O A$ und $O A^{\prime}$ sind. Die Oberfläche wird ein Kegel $\left.{ }^{*}\right)$, dessen Spitze $O^{\prime}$ und dessen Focallinien $O^{\prime} A^{\prime}$ und $B^{\prime} C^{\prime}$ sind. Dreht man das System der Linien $O A, O B$ mit unverändertem Winkel um $O$ nach $O A^{\prime}$ zu um den halben Winkel $\boldsymbol{A O A}^{\prime}$, so erhält man die beiden Linien, in denen der Kegel von der Hauptebene $A^{\prime} B^{\prime} C^{\prime}$ geschnitten wird, durch welche nebst den Brennlinien der Kegel bestimmt ist.

Wenn gleichzeitig $u=0, v=v$, erhält man

$$
\beta+\gamma=\alpha, \quad p^{2}=\frac{1}{4} v \text {. }
$$

Eine der Grössen $m^{2}$ und $n^{2}$ wird unendlich, während die andere endlich bleibt. Nimmt man an, dass $m$ unendlich wird, so giebt die aus den oben allgemein gefundenen Werthen folgende Gleichung

für den besonderen Fall

$$
\frac{m^{2} n^{2}}{m^{2}+n^{2}-p^{2}}=\frac{p^{2} \Delta^{2}}{\Delta^{2}-\Delta^{\prime 2}}
$$

$$
n^{2}=\frac{p^{2} \Delta^{2}}{\Delta^{2}-\Delta^{\prime 2}}=\frac{1}{4} \cdot \frac{v \Delta^{2}}{\Delta^{2}-\Delta^{\prime 2}}
$$

*) Es wird hierbei als bekannt vorausgesetzt, dass die Lage der Focallinien des Kegels, sowie derjenigen Linien, in welchen die erweiterte Ebene derselben den Kegel durchschneidet, eine derartige ist, dass sie dieselben Halbirungslinien haben, und dass jede zwei conjugirte Punkte auf diesen Linien von ihrem gemeinschaftlichen Schnittpunkte gleichweit entfernt sind.

H. 
Lässt man wieder $B$ und $C$ in $B^{\prime}$ und $C^{\prime}$ fallen, so ist $A A^{\prime}$ senkrecht auf $B^{\prime} C^{\prime}$; trifft $A A^{\prime}$ die Linie $B^{\prime} C^{\prime}$ in $H^{\prime}$, so hat man

und daher

$$
\begin{aligned}
& v=A H^{\prime 2}-A^{\prime} H^{\prime 2}, \\
& \frac{\Delta^{2}}{\Delta^{2}-\Delta^{\prime 2}}=\frac{A H^{\prime 2}}{v}
\end{aligned}
$$

$$
n^{2}=\frac{1}{4} A H^{\prime 2}
$$

Die Fläche ist ein Cylinder ${ }^{*}$ ), dessen Kante durch den Punkt $N^{\prime}$, die Mitte von $A^{\prime} H^{\prime}$, geht und mit $B^{\prime} C^{\prime}$ parallel ist. Die Basis des Cylinders wird ein Kegelschnitt, dessen Gleichung

$$
\frac{4 y^{2}}{A H^{\prime 2}}+\frac{4 z^{2}}{A H^{\prime 2}-A^{\prime} H^{\prime 2}}=1 \text {, }
$$

wo die Achse der $y$ senkrecht auf $B^{\prime} C^{\prime}$ steht und immer die grosse Achse der Basis des Cylinders wird, die eine Ellipse oder Hyperbel ist, jenachdem $A$ mehr oder weniger als $A^{\prime}$ von der Linie $B^{\prime} C^{\prime}$ entfernt ist. Die Punkte $N^{\prime}$ und $A^{\prime}, H^{\prime}$ werden der Mittelpunkt und die Brennpunkte dieser Basis. -

Die Fläche wird ein Ellipsoid, wenn $m^{2}, n^{2}, p^{2}$ positiv werden, also wenn $\mu, u, v, w$ positiv sind. Sie wird ein continuirliches Hyperboloid, wenn eine der Grössen $m^{2}, n^{2}, p^{2}$ negativ wird, während die andern positiv bleiben; also

a) wenn $m^{2}$ und $n^{2}$ positiv sind, also auch $\mu$ positiv ist, und wenn $p^{2}$ negativ ist, also $u, v, w$ negativ sind;

b) wenn $m^{2} n^{2}$ negativ ist, also $\mu$ negativ und $p^{2}$ positiv, also eine der Grössen $u, v, w$ negativ ist, während die beiden andern positiv sind, oder alle drei $u, v, w$ negativ sind, und von den Grössen $\sqrt{ }-u, \sqrt{ }-v, \sqrt{ }-w$ eine grösser als die Summe der beiden andern ist.

Die Fläche wird ein Hyperboloid mit zwei Flächen, wenn eine der Grössen $m^{2}, n^{2}, p^{2}$ positiv ist, und die beiden andern negativ werden; also wenn $m^{2} n^{2}$ und $p^{2}$ negativ werden, da $m^{2}$ und $n^{2}$, wie wir gesehen haben, nicht gleichzeitig negativ werden kőnnen. Dies erfordert die Bedingungen $\mu$ negativ und uvw positiv, also eine von ihnen positiv, die beiden andern negativ, oder es müssen alle drei positiv sein und eine der Grössen $\sqrt{ } u, \mathfrak{V} v, \sqrt{ } w$ grösser als die Summe der beiden andern.

*) In der That sind die Focallinien des Cylinders und diejenigen Linien, in denen die erweiterte Ebene derselben den Cylinder durchschneidet, zwei Systeme von parallelen Linien, deren gemeinschaftliche Mittellinie die Achse des Cylinders ist, und sind die Verbindungslinien jeder zwei conjugirten Punkte dieser Linien auf der Achse senkrecht.

$\boldsymbol{H}$. 
Als Grenze des Ellipsoids und zweiflächigen Hyperboloids erhält man das elliptische Paraboloid, wenn $u, v, w$ positiv sind und $\mu=0$.

Als Grenze des continuirlichen Hyperboloids erhält man das hyperbolische Paraboloid, wenn $u, v, w$ negativ sind und $\mu=0$.

Man kann dies in folgenden Satz zusammenfassen.

Satz.

„Es seien zwei Dreiecke $A B C$ und $A^{\prime} B^{\prime} C^{\prime}$ gegeben: in der Ebene von $A B C$ nehme man einen beliebigen Punkt $Q$ und construire über der Basis $A^{\prime} B^{\prime} C^{\prime}$ mit den Kanten $A^{\prime} P=A Q, B^{\prime} P=B Q, C^{\prime} P=C Q$ eine Pyramide: wenn der Punkt $Q$ sich in der Ebene des Dreiecks $A B C$ fortbewegt, beschreibt der Punkt $P$ eine Fläche der zweiten Ordnung, deren eine Hauptebene die Ebene der Basis $A^{\prime} B^{\prime} C^{\prime}$ ist, und deren eine Focalcurve dem Dreieck $A^{\prime} B^{\prime} C^{\prime}$ umschrieben ist.

Diese Fläche ist

1) Ein Ellipsoid, wenn alle Seiten des Dreiecks $A B C$ grösser als die entsprechenden Seiten des Dreiecks $A^{\prime} B^{\prime} C^{\prime}$ sind, und man mit den Seiten $\sqrt{B C^{2}-B^{\prime} C^{\prime 2}}, \sqrt{C A^{2}-C^{\prime} A^{\prime 2}}, \sqrt{A B^{2}-A^{\prime} B^{\prime 2}}$ ein Dreieck beschreiben kann.

2) Ein einflächiges Hyperboloid, wenn nur eine Seite des Dreiecks $A B C$ oder alle drei kleiner als die entsprechenden Seiten des Dreiecks $A^{\prime} B^{\prime} C^{\prime}$ sind.

3) Ein zweiflächiges Hyperboloid,

a) wenn eine Seite des Dreiecks $A B C$ grösser, die beiden andern kleiner als die entsprechenden Seiten des Dreiecks $A^{\prime} B^{\prime} C^{\prime}$ sind;

b) wenn alle Seiten des Dreiecks $A B C$ grösser als die entsprechenden Seiten des Dreiecks $A^{\prime} B^{\prime} C^{\prime}$ sind, man aber mit den Seiten

$$
\sqrt{B C^{2}-B^{\prime} C^{\prime 2}}, \quad \sqrt{C A^{2}-C^{\prime} A^{\prime 2}} \quad \sqrt{A B^{2}-A^{\prime} B^{\prime 2}}
$$

kein Dreieck construiren kann.

4) Ein elliptisches oder hyperbolisches Paraboloid, wenn die Seiten des Dreiecks $A B C$ alle grösser oder alle kleiner sind als die entsprechenden des Dreiecks $A^{\prime} B^{\prime} C^{\prime}$ und von den Quadratwurzeln der Differenzen der Quadrate der entsprechenden Seiten die eine gleich der Summe der beiden andern ist.

5) Eine Umdrehungsfläche, wenn die beiden Dreiecke $A B C$ und $A^{\prime} B^{\prime} C^{\prime}$ einander ähnlich sind, und zwar ist die Ebene der Basis $A^{\prime} B^{\prime} C^{\prime}$ selber immer die Hauptebene, welche Kreisschnitte giebt. 
6) Ein Kegel, wenn zwei entsprechende Seiten der Basen gleich sind.

7) Ein Cylinder, wenn ausserdem noch die Differenz der Quadrate der beiden andern Seiten gleich ist. ${ }^{66}$ -

Ich will jetzt den allgemeineren Fall behandeln, wo die Spitze der über der einen festen Basis errichteten Pyramide sich in einer beliebig gegebenen Ebene bewegt. Es sei $A B C$ die Projection dieser Basis auf die gegebene Ebene, und $h, i, k$ seien die Quadrate der Höhen ihrer Ecken über dieser Ebene. Denkt man sich die beiden Basen in solcher Lage, dass ihre Ecken drei Paare conjugirter Punkte zweier Ellipsoide sind, deren Gleichungen

$$
\frac{x x}{m m}+\frac{y y}{n n}+\frac{z z}{p p}=1, \quad \frac{x x}{m^{\prime} m^{\prime}}+\frac{y y}{n^{\prime} n^{\prime}}+\frac{z z}{p^{\prime} p^{\prime}}=1
$$

sind, so werden $A B C$ und die andere Basis $A^{\prime} B^{\prime} C^{\prime}$ in einer Hauptebene, der Ebene der $x$ und $y$, liegen und wieder die $x$-Coordinaten der entsprechenden Punkte der Dreiecke $A B C$ und $A^{\prime} B^{\prime} C^{\prime}$ sich wie $m: m^{\prime}$, die $y$-Coordinaten wie $n: n^{\prime}$ verhalten, woraus, wenn $O$ der Mittelpunkt der Fläche ist, wieder folgt, dass die Dreiecke $O B C, O C A, O A B$ sich wie die Dreiecke $O B^{\prime} C^{\prime}, O C^{\prime} A^{\prime}$, $O A^{\prime} B^{\prime}$ verhalten. Hieraus folgt ebenso wie oben, dass, um $O$ als Schwerpunkt sowohl von den Punkten $A, B, C$ wie von den Punkten $A^{\prime}, B^{\prime}, C^{\prime}$ zu erhalten, man in ihnen dieselben Gewichte anbringen muss. Nennt man diese Gewichte und ihre Summe wieder $\alpha, \beta, \gamma, \mu$ und $\Delta, A^{\prime}$ den Inhalt der Dreiecke $A B C$ und $A^{\prime} B^{\prime} C^{\prime}$, so hat man wieder ganz wie oben:

Es. wird ferner :

$$
\begin{aligned}
& O B C=\frac{\alpha}{\mu} A, \quad O C A=\frac{\beta}{\mu} A, \quad O A B=\frac{\gamma}{\mu} \Delta, \\
& O B^{\prime} C^{\prime}=\frac{\alpha}{\mu} \Delta^{\prime}, \quad O C^{\prime} A^{\prime}=\frac{\beta}{\mu} \Delta^{\prime}, \quad O A^{\prime} B^{\prime}=\frac{\chi}{\mu} \Delta^{\prime} .
\end{aligned}
$$

Nennt man wieder:

$$
O A^{2}+h-O A^{\prime 2}=O B^{2}+i-O B^{\prime 2}=O C^{2}+k-O C^{\prime 2}=p^{2} .
$$

$$
B C^{2}-B^{\prime} C^{\prime 2}=u, \quad C A^{2}-C^{\prime} A^{\prime 2}=v, \quad A B^{2}-A^{\prime} B^{\prime 2}=w,
$$

so hat man statt der früheren Gleichung folgende:

$$
\mu p^{2}+\frac{1}{\mu}(\beta \gamma u+\gamma \alpha v+\alpha \beta w)=\beta w+\gamma v+\mu h=\gamma u+\alpha v+\mu i=\alpha v+\beta u+\mu k \text {. }
$$

Setzt man den ersten Ausdruck $U$, so geben die drei Gleichungen

$$
\begin{aligned}
\alpha h+\beta(w+k)+\gamma(v+h) & =U, \\
\alpha(v+i)+\beta i+\gamma(u+i) & =U, \\
\alpha(v+k)+\beta(u+k)+\gamma k & =U
\end{aligned}
$$

die Werthe der drei Gewichte und ihrer Summe: 
und überdies

$$
\begin{aligned}
\alpha= & u(v+w-u)+(2 h-i-k) u-(i-k)(v-w), \\
\beta= & v(w+u-v)+(2 i-k-h) v-(k-h)(v-u), \\
\gamma= & w(u+v-w)+(2 k-h-i) w-(h-i)(u-v), \\
& \mu=2(v w+w u+u v)-u^{2}-v^{2}-w^{2}
\end{aligned}
$$

$$
U=2 u v w+h u(v+w-u)+i v(w+u-v)+k w(u+v-w) .
$$

Aus den Werthen von $\alpha, \beta, \gamma$ folgt

und daraus

$$
\begin{gathered}
\alpha \beta w+\gamma \alpha v=\alpha(U-\mu h), \\
\beta \gamma u+\alpha \beta w=\beta(U-\mu i), \\
\gamma \alpha v+\beta \gamma u=\gamma(U-\mu k)
\end{gathered}
$$

$$
2(\beta \gamma u+\gamma \alpha v+\alpha \beta v)=\mu\{U-(\alpha h+\beta i+\gamma k)\} .
$$

Es ist aber ursprünglich $U$ dem Ausdruck

$$
\mu p^{2}+\frac{1}{\mu}(\beta \gamma u+\gamma \alpha v+\alpha \gamma w)
$$

gleich gesetzt worden: man hat daher die Gleichung

woraus

$$
\boldsymbol{2}\left(U-\mu p^{2}\right)=U-(\alpha h+\beta i+\gamma k),
$$

$$
2 \mu p^{2}=U+h \alpha+i \beta+k \gamma
$$

und daher, wenn man die obigen Werthe von $\alpha, \beta, \gamma$ substituirt:

$$
\begin{aligned}
\mu p^{2}=u v w & +h u(v+w-u)+(h-i)(h-k) u \\
& +i v(w+u-v)+(i-k)(i-h) v \\
& +k w(u+v-w)+(k-h)(k-i) w .
\end{aligned}
$$

Man kann diesen Ausdruck auf merkwürdige Art transformiren. Man hat nämlich

$$
\begin{aligned}
\mu p^{2}= & u v w+v w(i+k)+w u(k+h)+u v(h+i) \\
& -h u^{2}-i v^{2}-k w^{2}+(h-i)(h-k) u+(i-k)(i-h) v+(k-h)(k-i) w \\
= & (u+i+k)(v+k+h)(w+h+i)-h u^{2}-i v^{2}-k w^{2} \\
& -2 h(i+k) u-2 i(k+h) v-2 k(h+i) w-(i+k)(k+h)(h+i) \\
= & (u+i+k)(v+k+h)(w+h+i) \\
& -h(u+i+k)^{2}-i(v+k+h)^{2}-k(w+h+i)^{2}+4 h i k .
\end{aligned}
$$

Setzt man jetzt

$$
u+i+k=u^{0}+2 \sqrt{i k}, \quad v+k+h=v^{0}+2 \sqrt{k h}, \quad w+h+i=w^{0}+2 \sqrt{h i},
$$

so wird

$$
\begin{aligned}
\mu p^{2}= & \left(u^{0}+2 \sqrt{\overline{i k}}\right)\left(v^{0}+2 \sqrt{k h}\right)\left(w^{0}+2 \sqrt{h i}\right) \\
& -h\left(u^{0}+2 \sqrt{\overline{i k}}\right)^{2}-i\left(v^{0}+2 \sqrt{k h}\right)^{2}-k\left(w^{01}+2 \sqrt{h i}\right)^{2}+4 h i k \\
= & u^{0} v^{0} w^{0}+2\left(\sqrt{\overline{i k}} \cdot v^{10} w^{0}+\sqrt{k h} \cdot w^{10} u^{0}+\sqrt{h i} \cdot u^{0} v^{0}\right)-h u^{102}-i v^{02}-k w^{02} .
\end{aligned}
$$


Die Grössen $u^{0}, v^{0}, w^{0}$ werden durch die Gleichungen

$$
\begin{aligned}
u^{0} & =u+(\sqrt{ } i-\sqrt{ } k)^{2}=B^{0} C^{02}-B^{\prime} C^{\prime 2}, \\
v^{0} & =v+(\sqrt{ } k-\sqrt{ } h)^{2}=C^{0} A^{02}-C^{\prime} A^{\prime 2}, \\
v^{0} & =v+(\sqrt{ } h-\sqrt{ } i)^{2}=A^{0} B^{02}-A^{\prime} B^{\prime 2}
\end{aligned}
$$

gegeben. Wenn $A^{0}$ ein Punkt des Ellipsoids ist, dessen Gleichung

$$
\frac{x^{2}}{m^{2}}+\frac{y^{2}}{n^{2}}+\frac{z^{2}}{p^{2}}=1 \text {, }
$$

und man mit dem Halbmesser $p$ eine concentrische Kugel beschreibt, so soll $D^{0}$ ein dem Punkte $A^{0}$ entsprechender Punkt der Kugel heissen, wenn die Coordinaten von $A^{0}$ aus den Coordinaten von $D^{0}$ dadurch erhalten werden, dass man sie mit $\frac{m}{p}, \frac{n}{p}, 1$ multiplicirt. Sind $D^{0}, E^{0}, F^{0}$ auf der Kugel den Punkten $A^{0}, B^{0}, C^{0}$ des Ellipsoids entsprechend, so hat man

$$
\begin{aligned}
u^{0} & =B^{0} C^{02}-B^{\prime} C^{\prime 2}=E^{0} F^{02}, \\
v^{0} & =C^{0} A^{02}-C^{\prime} A^{\prime 2}=F^{0} D^{02}, \\
w^{0} & =A^{0} B^{02}-A^{\prime} B^{\prime 2}=D^{0} E^{02} .
\end{aligned}
$$

Da die z-Coordinaten entsprechender Punkte des Ellipsoids und der Kugel gleich sind, so sind $h, i, k$ auch die Quadrate der Höhen von $D^{0}, E^{0}, F^{0}$ über der $x y$-Ebene. Nennt man ferner $D, E, F$ die Projectionen von $D^{0}, E^{0}, F^{0}$ auf die $x y$-Ebene, so wird

$$
\begin{gathered}
u=B C^{2}-B^{\prime} C^{\prime 2}=E F^{2}, \\
v=C A^{2}-C^{\prime} A^{\prime 2}=F D^{2}, \\
v=A B^{2}-A^{\prime} B^{\prime 2}=D E^{2} .
\end{gathered}
$$

Nennt man $r^{0}$ den Radius des dem Dreieck $D^{0} E^{0} F^{0}$ umschriebenen Kreises, so wird

es ist ferner

$$
16 r^{02} \cdot D^{0} E^{0} F^{02}=u^{0} v^{0} w^{0}
$$

$$
\mu=16 D E F^{2}=16 D^{0} E^{0} F^{02} \cos ^{2} \lambda,
$$

wenn $\lambda$ der Winkel ist, welchen die Ebenen der Dreiecke $D^{0} E^{0} F^{0}$ und $D E F$ mit einander bilden. Man hat daher

$$
p^{2} \mu-u^{0} v w^{0}=16 D^{0} E^{0} F^{02}\left(p^{2} \cos ^{2} \lambda-r^{02}\right),
$$

oder wenn man $\boldsymbol{H}$ das Quadrat des Perpendikels vom Mittelpunkt der Kugel auf die Ebene des Dreiecks $D^{0} E^{0} F^{0}$ nennt,

$$
\begin{aligned}
p^{2} \mu-u^{0} v^{0} w^{0} & =16 D^{0} E^{0} F^{02}\left(H \cos ^{2} \lambda-r^{02} \sin ^{2} \lambda\right) \\
& =2\left(\sqrt{i k} \cdot v^{0} w^{0}+\sqrt{k h} \cdot w^{0} u^{0}+\sqrt{h i} \cdot u^{0} v^{0}\right)-\left(h u^{02}+i v^{02}+k v^{02}\right) .
\end{aligned}
$$


Nennt man $h^{0}, i^{0}, k^{0}, \mathfrak{H}$ die Quadrate der Perpendikel, die von $\boldsymbol{D}^{0}$, $E^{0}, F^{0}$ und vom Mittelpunkte des dem Dreieck $D^{0} E^{0} F^{0}$ umschriebenen Kreises auf die Schneidelinie beider Ebenen gefält werden, so wird

$$
\mathfrak{H}=\boldsymbol{H} \cdot \operatorname{ctg}^{2} \lambda, \quad h^{0}=\frac{h}{\sin ^{2} \lambda}, \quad i^{0}=\frac{i}{\sin ^{2} \lambda}, \quad k^{0}=\frac{k}{\sin ^{2} \lambda} ;
$$

wenn man daher die vorige Gleichung durch $\sin ^{2} \lambda$ dividirt, erhält man $16 D^{0} E^{0} F^{02}\left(\mathfrak{H}-r^{02}\right)=16 D^{0} E^{0} F^{02} \cdot \mathfrak{H}-u^{0} v^{0} w^{0}$

$$
=\left\{\begin{array}{l}
2\left(\sqrt{i^{0} k^{10}} \cdot v^{0} w^{0}+\sqrt{k^{0} h^{0}} \cdot w^{0} u^{0}+\sqrt{h^{0 i^{0}}} \cdot u^{0} v^{0}\right) \\
-h^{0} \cdot u^{02}-i^{01} \cdot v^{02}-k^{0} \cdot w^{02}
\end{array}\right.
$$

Auf diese Weise lässt sich der für $\mu p^{2}$ gefundene Ausdruck auf eine Formel der algebraischen Geometrie zurūckführen, indem in der vorstehenden

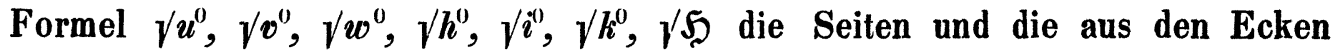
und dem Mittelpunkt des umschriebenen Kreises auf eine beliebige Linie gefällten Perpendikel bedeuten. Die Grösse $\mathfrak{S}-r^{02}$ ist das Quadrat der Tangente, die man an den Kreis vom Fusspunkte des Perpendikels $\sqrt{ } /$ f fältt. Man sieht leicht, dass man in der Formel $\sqrt{ } \mathfrak{S}, \sqrt{ } h^{0}, \sqrt{ } i^{0}, \sqrt{ } k^{0}$ um dieselbe Grösse ändern kann, welches darauf hinaus kommt, dass man die gerade Linie parallel mit sich verrückt. Man hat nämlich

$$
16 D^{0} E^{0} F^{02}=2\left(v^{0} w^{0}+w^{0} u^{0}+u^{0} v^{0}\right)-u^{02}-v^{02}-w^{02}
$$

und nach einem bekannten Satze vom Schwerpunkt

$$
16 D^{0} E^{0} F^{02} \cdot / \mathfrak{S}=u^{0}\left(v^{0}+w^{0}-u^{0}\right) / h^{0}+v^{0}\left(w^{0}+u^{0}-v^{0}\right) V^{0}+w^{0}\left(u^{0}+v^{0}-w^{0}\right) V^{1} k^{0},
$$

da der Mittelpunkt des umschriebenen Kreises der Schwerpunkt der Punkte $D_{0}, E_{0}, F_{0}$ ist, wenn man in ihnen die Coefficienten von $\sqrt{ } h^{0}, \sqrt{ } i^{0}, \sqrt{ } k^{0}$ als Gewichte anbringt, und die Summe dieser Gewichte gleich $16 D^{0} E^{0} F^{02}$ ist. Wenn man die erste Gleichung mit $\left(\sqrt{ } \mathfrak{S}-r^{0}\right)^{2}$, die zweite mit $-2\left(\sqrt{ } \mathfrak{S}-r^{0}\right)$ multiplicirt zu der gefundenen Formel hinzufügt ${ }^{*}$ ) und

$$
\sqrt{ } h^{0}-\sqrt{ } \mathfrak{S}+r^{0}=\sqrt{ } h^{\prime}, \quad \sqrt{ } i^{0}-\sqrt{ } \mathfrak{S}+r^{0}=\sqrt{ } i^{\prime}, \quad \sqrt{ } k^{0}-\sqrt{ } \mathfrak{S}+r^{0}=\sqrt{ } k^{\prime}
$$

setzt, so erhält man

$$
0=2\left(\sqrt{i^{\prime} k^{\prime} \cdot v^{0} w^{0}}+\sqrt{k^{\prime} h^{\prime}} \cdot w^{0} u^{0}+\sqrt{h^{\prime} i^{\prime} \cdot} \cdot u^{0} v^{0}\right)-h^{\prime} u^{02}-i^{\prime} v^{02}-k^{\prime} w^{02},
$$

wo $\sqrt{ } h^{\prime}, V^{\prime} i^{\prime}, \gamma^{\prime} k^{\prime}$ die auf eine Tangente des dem Dreieck $D^{0} E^{0} F^{\prime \prime}$ umschriebenen

*) Das Ergebniss dieser Rechnung kommt damit überein, die Lothe $\sqrt{\mathfrak{S}}, \sqrt{h^{0}}, \sqrt{ } \mathbf{i}^{0}$, $\sqrt{ } k^{0}$ um $\sqrt{ } \mathfrak{S}-r^{0}$ zu verkürzen, oder, was dasselbe ist, die auf diesen Lothen senkrechte gerade Linie parallel mit sich selbst so zu verschieben, das sie eine Tangente des Kreises um $D^{0} E^{0} F^{0}$ wird. 
Kreises aus $D^{0}, E^{0}, F^{0}$ gefällten Perpendikel bedeuten. Aus der vorstehenden Gleichung folgt, dass von den Quadratwurseln aus den drei Grössen $u^{0} \sqrt{ } h^{\prime}$, $v^{0} \sqrt{i}^{\prime}, w^{0} \sqrt{ } k^{\prime}$ oder von den drei Grössen $E^{0} F^{0}{ }^{4} k^{\prime}, F^{0} D^{0}{ }^{4} i^{\prime}, D^{0} E^{0}{ }^{4} k^{\prime}$ eine die Summe der beiden anderen ist. Man kann die Grössen $\sqrt{ }^{4} h^{\prime},{ }^{4} i^{\prime},{ }^{4} k^{\prime}$ leicht construiren. Nennt man nämlich $T$ den Berührungspunkt, so wird nach bekannten Sätzen der Elementargeometrie

$$
\sqrt{2 r^{0} \cdot \sqrt{ } h^{\prime}}=D^{0} T, \quad \sqrt{2 r^{0} \cdot \sqrt{i^{\prime}}}=E^{0} T, \quad \sqrt{2 r^{0} \cdot \sqrt{ } h^{\prime}}=F^{0} T,
$$

so dass die drei Grössen

$$
E^{0} F^{0} \cdot \sqrt{2 r^{0} \cdot \sqrt{ } k^{\prime}}, \quad F^{0} D^{0} \cdot \sqrt{2 r^{0} \cdot \sqrt{i^{\prime}},} \quad D^{0} E^{0} \cdot \sqrt{2 r^{0} \cdot \sqrt{ } k^{\prime}}
$$

die drei Producte der gegenüberstehenden Seiten und Diagonalen in dem dem Kreise eingeschriebenen Viereck $D^{0} E^{v} F^{0} T$ sind, von denen bekannt ist, dass die Summe von zweien dem dritten gleich ist. Unsre Formel für $p^{2}$ wird so auf den sogenannten Ptolemäischen Lehrsatz zurückgeführt, welcher andererseits in dieser Form ausgesprochen durch das Vorstehende eine grosse Ausdehnung erhält.

Wie in dem früheren Falle findet man $m$ und $n$ aus den beiden Gleichungen

$$
\begin{aligned}
& \frac{m n}{p^{2}}=\frac{A B C}{D E F}=\frac{4 \Delta}{\sqrt{\mu}}, \\
& \frac{m^{\prime} n^{\prime}}{p^{2}}=\frac{A^{\prime} B^{\prime} C^{\prime}}{D E F}=\frac{4 \Delta^{\prime}}{\sqrt{\mu}} .
\end{aligned}
$$

Man sieht aus diesen Gleichungen, dass $m^{2} n^{2}$ und $m^{\prime 2} n^{\prime 2}$ dasselbe Zeichen wie $\mu$ haben, dass also der $x y$-Schnitt eine Ellipse oder Hyperbel ist, jenachdem $\mu$ positiv oder negativ ist. Die Fläche wird nur dann ein einflächiges Hyperboloid, wenn $m^{2} n^{2} p^{2}$ negativ ist, also wenn $\mu$ und $p^{2}$ verschiedene Zeichen haben. Die Werthe $\frac{m}{p}, \frac{n}{p}$ werden ganz ebenso, wie im. einfacheren Fall, durch die Seiten der Dreiecke $A B C$ und $A^{\prime} B^{\prime} C^{\prime}$ ausgedrückt, und nur $p^{2}$ wird verschieden. Mit Hilfe des früheren Hilfsdreiecks $D E F$ kann man die Grösse $p^{2}$ so construiren: Man errichte in den Punkten $D, E, F$ die Perpendikel $\sqrt{ } h, \sqrt{ } i, \sqrt{ } k$, deren Endpunkte $D^{0}, E^{0}, F^{0}$ seien, ferner errichte man in dem Mittelpunkt des dem Dreieck $D^{0} E^{0} F^{0}$ umschriebenen Kreises ein Perpendikel auf der Ebene $D^{0} E^{0} F^{0}$ und bestimme den Punkt, in welchem dies Perpendikel die Ebene $D E F$ durchschneidet, dann wird $p$ die Entfernung dieses Punktes von jedem der Eckpunkte $D^{0}, E^{0}, F^{0}$, (oder, was dasselbe ist, $p$ wird der Radius derjenigen dem Dreieck $D^{0} E^{0} F^{0}$ umschriebenen Kugel, deren Mittelpunkt in der Ebene DEF liegt). 